\title{
Analytical, Circle-to-Circle Low-Thrust Transfer Trajectories with Plane Change
}

\author{
Malcolm Macdonald ${ }^{1}$, \\ Advanced Space Concepts Laboratory, Department of Mechanical \& Aerospace Engineering, \\ University of Strathclyde, Glasgow, G1 1XQ, Scotland
}

Orbit averaging techniques are used to develop analytical approximations of circle-tocircle low-thrust trajectory transfers with plane-change about the Sun. Separate expressions are developed for constant acceleration, or thrust, electric propulsion, solar sail propulsion and combined, or hybrid electric (constant acceleration or thrust) / solar sail propulsion. The analytical expressions uniquely allow the structure of circle-to-circle low-thrust trajectory transfers with plane-change about the Sun to be understood, and the optimal trajectory structure is analytically derived for each propulsion system considered. It is found that the optimal fixed thrust electric propulsion transfer reduces the orbit radius with no plane change and then performs the plane-change, while the optimal solar sail and hybrid transfers combine the reduction of orbit radius with some plane change, before then completing the plane change. The optimal level of plane change during the reduction of orbit radius is derived and it is found the analytically-derived minimum time solar sail transfer is within $1 \%$ of the numerically-derived optimal transfer. It is also found that, under the conditions considered, a sail characteristic acceleration of less than $0.5 \mathrm{~mm} / \mathrm{s}^{2} \mathrm{can}$, in 5 -years, attain a solar orbit that maintains the observer-to-solar pole zenith angle below 40 degrees for 25 days; the approximate sidereal rotation period of the Sun. However, a sail characteristic acceleration of more than $0.5 \mathrm{~mm} / \mathrm{s}^{2}$ is required to attain an observer-to-solar pole zenith angle below 30 degrees for 25 days within 5-years of launch. Finally, it was found that the hybridization of electric propulsion and solar sail propulsion was, typically, of more benefit when the system was thrust constrained than when it was mass constrained.

\section{Nomenclature}

\begin{tabular}{|c|c|c|}
\hline \multicolumn{2}{|c|}{ Latin Letters } & \\
\hline$A_{\text {sail }}$ & $=$ & Sail surface area, $\mathrm{m}^{2}$ \\
\hline$a$ & $=$ & Semi-major axis, $m$ (unless otherwise stated) \\
\hline$c$ & $=$ & propulsion system exhaust velocity, $\mathrm{m} / \mathrm{s}$ \\
\hline$i$ & $=$ & inclination, $\mathrm{rad}$ (unless otherwise stated) \\
\hline$m$ & $=$ & Mass of spacecraft, $\mathrm{kg}$ \\
\hline$N$ & $=$ & Spacecraft centered frame of reference, normal direction \\
\hline $\mathrm{N}_{0}$ & $=$ & Defined in Eq. (38) \\
\hline $\mathrm{N}_{1}$ & $=$ & Defined in Eq. (39) \\
\hline$R$ & $=$ & Spacecraft centered frame of reference, radial direction \\
\hline$r$ & & Orbit radius, $m$ (unless otherwise stated) \\
\hline $\mathbf{T}$ & & Thrust, $N$ (unless otherwise stated) \\
\hline$T$ & $=$ & Spacecraft centered frame of reference, tangential direction \\
\hline & $=$ & Time, $s$ (unless otherwise stated) \\
\hline
\end{tabular}

Greek Letters

$\alpha \quad=$ Solar sail cone angle, $\mathrm{rad}$

$\beta \quad=$ Solar sail lightness number

\footnotetext{
${ }^{1}$ Associate Director, Advanced Space Concepts Laboratory, Mechanical \& Aerospace Engineering, University of Strathclyde, Glasgow, G1 1XQ, Scotland. AIAA Associate Fellow.
}

1

American Institute of Aeronautics and Astronautics 

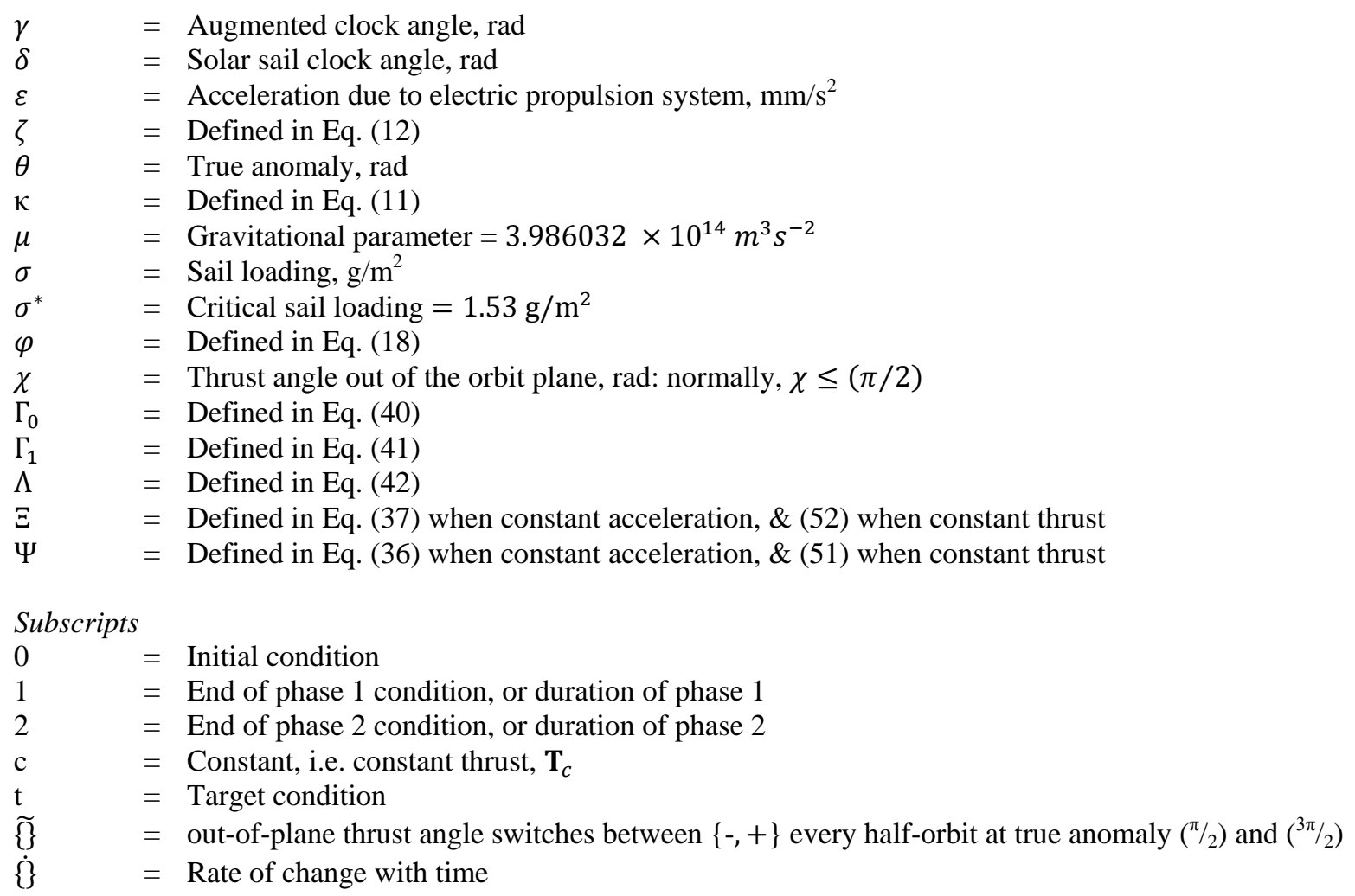

\section{Introduction}

$\mathrm{T}$

HE mission concept to place a spacecraft into an high-inclination, or polar orbit over the Sun using solar sail propulsion has been extensively studied since the nineteen seventies. ${ }^{1-5}$ From the earliest studies, it has been clear that such a mission is technically challenging and, in most likelihood, requires the maturation of a form of lowthrust propulsion currently considered as advanced, such as solar sailing. ${ }^{6}$ Prior studies have relied on numerical optimization techniques to derive the time optimal transfer trajectory of the solar sail propelled spacecraft. ${ }^{1-5,7-10}$ Consequently, the structure of the solar sail time optimal transfer trajectory has been deduced, and is relatively wellunderstood. It should be noted that within these prior studies both ideal and non-ideal solar sail propulsion models ${ }^{6}$ and even the effect of optical surface degradation ${ }^{11,12}$ have been considered.

This paper presents, for the first time, a fully analytical derivation of an approximately time-optimal transfer from a circular orbit, within the Ecliptic plane, at 1 astronomical unit, au, to a circular, high-inclination orbit of the Sun at radius less than, or equal to 1 au. Transfers are derived for solar sail propulsion, electric propulsion, and hybrid solar sail / electric propulsion, and as such, the techniques developed should be extendable to any other form of low-thrust propulsion. By deriving analytical solutions to the approximately time-optimal transfer unique insights to the problem can be gained, allowing comparison of the optimal transfer trajectory structures using different propulsion systems, and, for the first time allow previously derived solutions to be validated. Additionally, the use of an analytical derived solution allows technology trades to be performed significantly quicker than would otherwise be possible, for example if using any form of numerical optimization.

\section{Mission Architecture}

In many previous solar polar mission concepts, ${ }^{1-5,7-10}$ the mission science goals required an Earth resonant orbit, placing the spacecraft near to the solar limb as seen from Earth to allow observation of the corona along the Sun-Earth line. An Earth resonant orbit limits the orbit radius to $N^{-2 / 3}$ au, where $N$ is an integer representing the resonant value. If this science requirement is not present then the only other rational to adopt a resonant orbit would be to allow continuous spacecraft visibility from Earth for ease of spacecraft operations. It is of note that in mission concepts that do not require a resonant orbit, a resonant orbit radius has typically still been chosen. ${ }^{13}$

The peak-duration of communication blackouts due to solar conjunctions against a range of orbit radius for both $\mathrm{X}$-band and K-band communications is shown in Fig. 1 assuming perpendicular relative motion. Note that a 4- 
degree field-of-view exclusion is assumed for X-band communications, while a 1.5-degree field-of-view exclusion is assumed for K-band communications. It is seen from Fig. 1 that the likely peak-duration of communication blackouts, on a solar polar orbit, due to solar conjunctions is around two-days for X-band systems and less than 1day for K-band systems.

\section{A. Transfer Trajectory}

The transfer trajectory to a high-inclination, circular orbit will assume a two-phase approach, whereby the orbit radius will be restricted to greater than or equal to the target orbit radius in an attempt to minimize the technology requirements across the complete spacecraft system. The two phases are, reduction of orbit radius to the target value, whilst perhaps performing some plane change maneuver at the same time, and secondly, the rapid increase of orbit inclination to target value while maintaining a near-constant orbit radius.

It has been previously seen that for solar sail transfers to such high-inclination, circular orbits that the first phase should include some amount of plane change. ${ }^{1-5,7-10}$ However, the optimal level of plane-change has not previously been detailed. Furthermore, to fully time-optimize a solar sail transfer to a high-inclination orbit, it has previously been seen that the orbit radius at which the orbit plane change maneuver is performed should not be restricted to the target orbit radius. ${ }^{1-5,7-10}$ That is, orbit inclination is changed more rapidly by reducing the orbit radius below the target orbit radius, introducing a third phase where the orbit radius is increased back to the target value, whist perhaps also performing a small amount of plane change. It has been shown that such a three-phase transfer can also be used to minimize the technology requirements on the solar sail. ${ }^{4}$ Specifically, for fixed transfer duration, the required sail size can be minimized by correctly defining the orbit radius in phase 2 . As this radius is reduced the spacecraft size increases due to the thermal loading on the spacecraft, however the sail performance also increases due to the $\left(1 / r^{2}\right)$ variation in sail performance, reducing the required sail size for a fixed transfer duration. In [4] it is shown that a turning point can be defined at which the mass penalty due to the thermal loadings overcomes the increase in sail performance, this turning point defines the minimum sail technology requirement design point. However, the analysis in [4] does not directly consider spacecraft or mission cost, or risk, and it is likely that the trajectory profile for a minimum cost / risk mission would increase the phase 2 radius above both the time optimal and sail technology optimal radius. Indeed assuming the target orbit radius is less than that of, say, Venus, it is likely that the minimum cost mission would result in the removal of phase three, with phase two occurring at the target orbit radius and hence the spacecraft thermal design load would be the target/operational orbit radius and not that during the transfer trajectory.

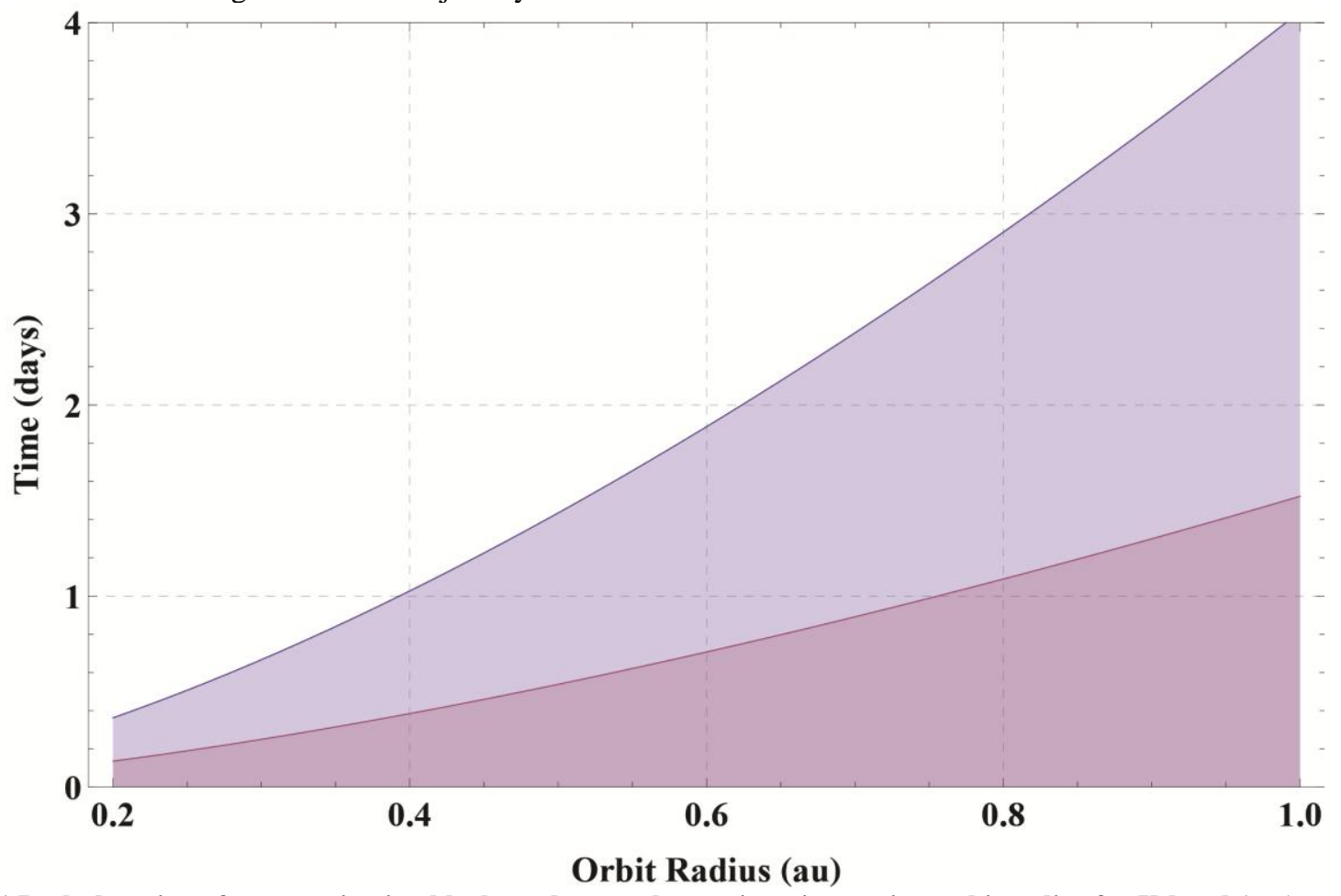

Fig. 1 Peak-duration of communication blackout due to solar conjunction against orbit radius for X-band (top) and Kband (bottom) communication, assuming perpendicular motion.

American Institute of Aeronautics and Astronautics 


\section{Electric Propulsion General Perturbations Approximation}

A low-thrust orbit maneuver from an initially, or nearly circular orbit, using tangential thrusting can be approximated as a quasi-circular spiral ${ }^{14}$ as such the orbit radius, $r$, can be used to replace the semi-major axis, assuming that eccentricity is negligible. Note that with tangential thrusting although the instantaneous variation in eccentricity is non-zero, the secular variation of eccentricity is zero. It is noted from Eq. (1) that any component of thrust that is out of the orbit plane will not contribute towards any orbit raising / lowering, i.e. energy change, maneuver as only in-plane thrusting alters the rate of change of orbit semi-major axis. Resultantly, within this analytical analysis the spacecraft thrust is directed along the vehicle tangential direction and pitched at an angle $\chi$ out of the orbit plane, as shown in Fig. 2, such that the orbit energy and plane can be controlled. The radial component of acceleration is thus zero. The Lagrange-Gauss variational equations for semi-major axis, inclination, and true anomaly become, ${ }^{15}$

$$
\begin{aligned}
& \frac{d r}{d t}=\frac{d a}{d t}=\frac{2}{\sqrt{\mu}} \frac{|\mathbf{T}|}{m(t)} \cos \chi a^{\frac{3}{2}} \\
& \frac{d i}{d t}=\frac{|\mathbf{T}|}{m(t)} \sin \chi \cos \theta \sqrt{\frac{a}{\mu}} \\
& \frac{d \theta}{d t}=\sqrt{\frac{\mu}{a^{3}}}
\end{aligned}
$$

$m(t)=m_{0}-\dot{m} t$

The Lagrange-Gauss variational equations may be averaged with respect to true anomaly to obtain the longperiod motion using the averaging operator,

$$
\langle z\rangle=\frac{1}{2 \pi} \int_{0}^{2 \pi} z d \theta
$$

gaining,

$$
\begin{aligned}
& \left\langle\frac{d a}{d t}\right\rangle=\frac{a^{\frac{3}{2}}}{\pi \sqrt{\mu}} \frac{|\mathbf{T}|}{m(t)} \int_{0}^{2 \pi} \cos \chi(\theta) d \theta \\
& \left\langle\frac{d i}{d t}\right\rangle=\frac{1}{2 \pi} \frac{|\mathbf{T}|}{m(t)} \sqrt{\frac{a}{\mu}} \int_{0}^{2 \pi} \sin \chi \cos \theta(\theta) d \theta
\end{aligned}
$$

It should be noted that the use of orbit averaging to obtain long-period motion means that any orbit maneuver will only be accurately represented over an integer number of revolutions.

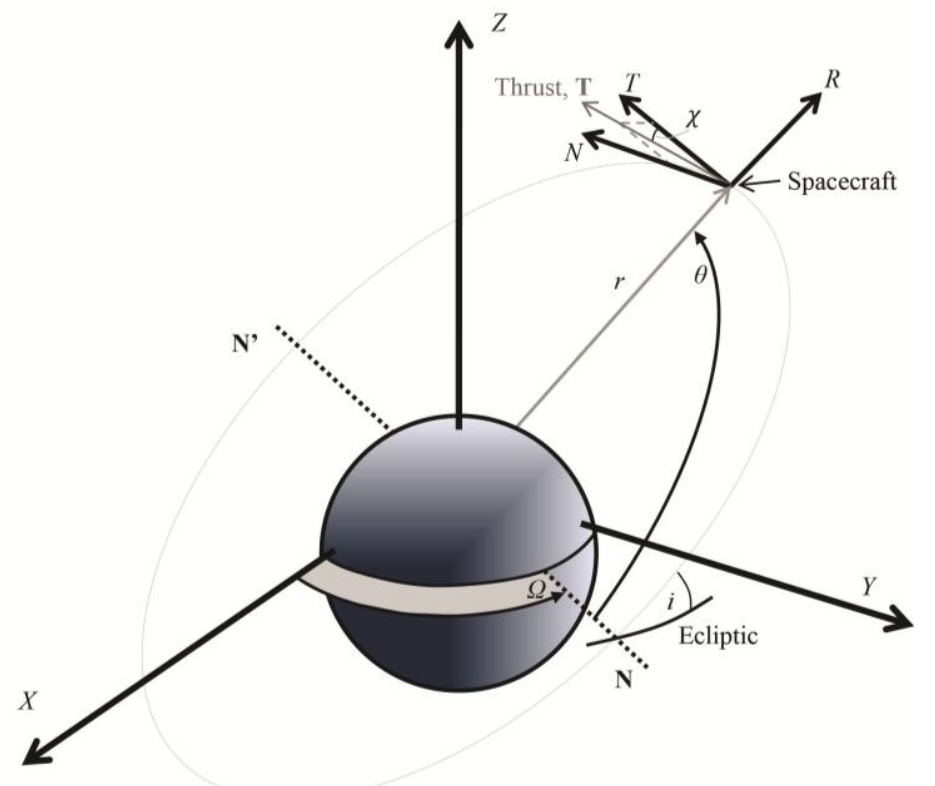

Fig. 2 Orbit geometry with ascending node $\mathrm{N}$ and descending node $\mathrm{N}$ ' and thrust induced acceleration orientation 4

American Institute of Aeronautics and Astronautics 


\section{A. Constant Acceleration}

For a spacecraft with low-thrust propulsion, such as a Solar Electric Propulsion (SEP) system, the reduction in spacecraft mass due to exhaust gases may, in-general, be neglected for short duration maneuvers. ${ }^{16,17}$ As such systems generally maintain constant thrust, this leads the initial acceleration, $\varepsilon_{0}$, to remain constant. Equations (6) (7) may be re-written using Eq. (8) and assuming zero mass change with respect to time.

$$
\frac{|\mathbf{T}|}{m(t)}=\frac{\varepsilon_{0}}{1-\frac{\varepsilon_{0}}{c} t}
$$

where, $c$ is the propulsion system exhaust velocity. Thereafter, the modified Eq. (6) - (7) can be solved by defining an out-of-plane thrust angle profile with respect to true anomaly. From Eq. (2) it can be seen that for a fixed out-ofplane angle $\chi(\neq 0, \pi, 2 \pi)$ the rate of change of inclination will switch sign at true anomaly $(\pi / 2)$ and $(3 \pi / 2)$. Therefore, by switching the out-of-plane thrust vector orientation in accordance with this variation, a uniformly positive or negative variation in inclination can be gained. ${ }^{18-20}$ Equation (6) is thus solved directly to obtain,

$$
a_{1}=\frac{a_{0}}{\left(1 \mp \varepsilon_{0} \cos \tilde{\chi} \sqrt{\frac{a_{0}}{\mu}} t\right)^{2}}
$$

where, $\tilde{\chi}$ switches between $\{-\chi,+\chi\}$ every half-orbit at true anomaly $(\pi / 2)$ and $(3 \pi / 2)$ and the ' $\mp$ ' term is used for a positive or negative rate of change of semi-major axis, respectively. Equation (7) can thereafter be solved sequentially to obtain,

$$
i(t)=i_{0}+\kappa \zeta
$$

where,

$$
\begin{aligned}
& \zeta=\log \left(1 \mp \varepsilon_{0} \cos \tilde{\chi} \sqrt{\left.\frac{a_{0}}{\mu} t\right)}\right. \\
& \kappa=\mp\left(\frac{2}{\pi}\right) \tan \tilde{\chi}
\end{aligned}
$$

with the ' $\mp$ ' term used for a positive or negative rate of change of semi-major axis, respectively. Using Eq. (9) and (10) it can be shown that for $\tilde{\chi}=80.8112^{\circ}$ a low-thrust propelled spacecraft will transfer from a circular 1 au orbit within the ecliptic plane to a circular 0.48 au orbit over the solar poles, inclination 82.75 degrees, in $\left(2.62058 \times 10^{-3} / \varepsilon_{0}\right)$ years. However, it is noted that whilst a coupled plane change and orbit energy manoeuvre is possible it is likely to be considered non-ideal from a science perspective, and as will be seen, such a trajectory is also sub-optimal in time and fuel mass consumption.

Equation (9) can be re-written to give the first phase duration, assuming a negative change in semi-major axis, for a given out-of-plane angle, as,

$$
t_{1}=\frac{\sec \tilde{\chi}\left(\sqrt{\mu} a_{0}-\mu \sqrt{\frac{a_{0}}{\mu}} \sqrt{a_{1}}\right)}{\varepsilon_{0} a_{0} \sqrt{a_{1}}}
$$

with Eq. (10) thereafter giving the orbit inclination at the end of the first phase. To find the time required for the second phase to complete the required orbit plane change Eq. (10) must be re-derived for $\chi=(\pi / 2)$. Noting that for $\beta=(\pi / 2)$ the change in semi-major axis will be zero, Eq. (10) becomes,

$$
i(t)=i_{1}+2 \frac{\varepsilon_{0}}{\pi} \sqrt{\frac{a_{1}}{\mu}} t
$$

hence, the second phase duration is,

$$
t_{2}=\frac{\pi}{2} \frac{i_{t}-i_{1}}{\varepsilon_{0} \sqrt{a_{1} / \mu}}
$$

where, $i_{t}$ is the target orbit inclination and the total orbit transfer duration is $t=\left(t_{1}+t_{2}\right)$.

\section{B. Constant Thrust}

The derivation of Eq. (9) and (10) assumed that a constant acceleration was delivered by the low-thrust propulsion system. However, this result can be extended to consider constant thrust from the low-thrust propulsion system, where reduction in spacecraft mass due to exhaust gases is no longer neglected, hence providing a more accurate solution. Using Eq. (8) for only constant thrust to rewrite Eq. (6) and subsequently solving gives, 


$$
a_{1}=\frac{a_{0}}{\left(1 \pm \sqrt{\frac{a_{0}}{\mu}} c \log \left(1-\frac{t \varepsilon_{0}}{c}\right) \cos \chi\right)^{2}}
$$

here, $\tilde{\chi}$ switches between $\{-\chi,+\chi\}$ every half-orbit, with the ' \pm ' term being used for a positive or negative rate of change of semi-major axis, respectively. Similarly, Eq. (7) can thereafter be solved to obtain,

$$
i(t)=i_{0}+\kappa \varphi
$$

where, $\kappa$ is as shown in Eq. (12), and

$$
\varphi=\log \left[1 \pm c \sqrt{\frac{a_{0}}{\mu}} \cos \tilde{\chi} \log \left(1-\frac{\varepsilon_{0} t}{c}\right)\right]
$$

with the ' \pm ' and ' $\mp$ ' term being used for a positive or negative rate of change of semi-major axis, respectively. Equation (16) can be re-written to give the first phase duration, assuming a negative change in semi-major axis, for a given out-of-plane angle, as,

$$
t_{1}=\frac{c}{\epsilon_{0}} \exp \left(-\frac{\sqrt{\mu} \sec \tilde{\chi}}{c \sqrt{a_{1}}}\right)\left[\exp \left(\frac{\sqrt{\mu} \sec \tilde{\chi}}{c \sqrt{a_{1}}}\right)-\exp \left(\frac{\sqrt{\mu} \sec \tilde{\chi}}{c \sqrt{a_{0}}}\right)\right]
$$

with Eq. (17) thereafter giving the orbit inclination at the end of the first phase.

To find the time required for the second phase to complete the required orbit plane change Eq. (17) must be rederived for $\chi=(\pi / 2)$. Noting that for $\chi=(\pi / 2)$ the change in semi-major axis will be zero, Eq. (17) becomes,

$$
i_{1}=i_{0} \mp \frac{2}{\pi} c \sqrt{\frac{a}{\mu}} \log \left(1-\frac{t \varepsilon_{0}}{c}\right)
$$

hence, the second phase duration is,

$$
t_{2}=\frac{c}{\varepsilon_{0}}\left(-1+e^{\frac{\left( \pm i_{0} \mp i_{1}\right) \pi \sqrt{\mu}}{2 \sqrt{a} c}}\right)
$$

\section{Solar Sail General Perturbations Approximation}

The prior analysis can be repeated for a solar sail propelled spacecraft. Once again assuming an initially, or nearly circular orbit, the Lagrange-Gauss variational equations presented in Eqs. (1) - (3) can be written,

$$
\begin{aligned}
& \frac{d r}{d t}=\frac{d a}{d t}=\frac{2}{\sqrt{\mu}} a^{\frac{3}{2}} T \\
& \frac{d i}{d t}=\sqrt{\frac{a}{\mu}} \cos \theta N \\
& \frac{d \theta}{d t}=\sqrt{\frac{\mu}{a^{3}}}
\end{aligned}
$$

and averaged using Eq. (5) to give,

$$
\begin{aligned}
& \left\langle\frac{d a}{d t}\right\rangle=\frac{a^{\frac{3}{2}}}{\pi \sqrt{\mu}} \int_{0}^{2 \pi} T(\theta) d \theta \\
& \left\langle\frac{d i}{d t}\right\rangle=\frac{1}{2 \pi} \sqrt{\frac{a}{\mu}} \int_{0}^{2 \pi} \cos \theta(\theta) N d \theta
\end{aligned}
$$

From [6] the tangential and out-of-plane perturbation due to the solar sail acceleration is,

$$
\begin{aligned}
& T=\beta \frac{\mu}{a^{2}} \cos \alpha^{2} \sin \alpha \sin \delta \\
& N=\beta \frac{\mu}{a^{2}} \cos \alpha^{2} \sin \alpha \cos \delta
\end{aligned}
$$

where, $\beta$ is the sail lightness number, $\alpha$ is the sail pitch angle and $\delta$ is the sail clock angle as defined in [6].

From $[6,18-20]$ the locally optimal (positive) rate of change of semi-major axis / radius, and hence orbit energy, is achieved when $\alpha=\tan ^{-1}(1 / \sqrt{2})$ and $\delta=(\pi / 2)$; for a negative rate of change $\delta=(3 \pi / 2)$. And, from $[6,18-20]$ the locally optimal rate of change of inclination is achieved when $\alpha=\tan ^{-1}(1 / \sqrt{2})$ and $\delta=\{0, \pi\}$, with the switch of clock angle occurring as for the electric propulsion case at true anomaly $\pi / 2$ and $3 \pi / 2$. 
In order to aid the analysis an augmented clock angle is defined, measured from the transverse axis, such that $\gamma=(\pi / 2-\delta)$; meaning that the augmented clock angle, $\gamma$, will switch sign at true anomaly $(\pi / 2)$ and $(3 \pi / 2)$ when considering the change of orbit inclination.

Combining Eq. (25) and (27), gives,

$$
\left\langle\frac{d a}{d t}\right\rangle=\frac{\beta}{\pi} \sqrt{\frac{\mu}{a}}(\cos \alpha)^{2} \sin \alpha \int_{0}^{2 \pi} \sin \left(\frac{\pi}{2}-\tilde{\gamma}\right)(\theta) d \theta
$$

where, $\tilde{\gamma}$ switches between $\{-\gamma,+\gamma\}$ every half-orbit at true anomaly $(\pi / 2)$ and $(3 \pi / 2)$. Noting that $\sin \left(\frac{\pi}{2}-\vartheta\right)=\cos \vartheta$ and $\sin \left(\frac{3 \pi}{2}-\vartheta\right)=-\cos \vartheta$, and integrating,

$$
\left\langle\frac{\mathrm{d} a}{\mathrm{~d} t}\right\rangle= \pm 2 \beta \sqrt{\frac{\mu}{a}}(\cos \alpha)^{2} \sin \alpha \cos \tilde{\gamma}
$$

where, $0 \leq \tilde{\gamma} \leq(\pi / 2)$ with the ' \pm ' term is used for a positive or negative rate of change of semi-major axis. Direct integration yields,

$$
\mathrm{t}=\mp \frac{\left(a_{1}^{3 / 2}-a_{0}^{3 / 2}\right)}{3 \sqrt{\mu} \beta(\cos \alpha)^{2} \sin \alpha \cos \tilde{\gamma}}
$$

where, again the ' $\mp$ ' term is used for a positive or negative rate of change of semi-major axis respectively. That is, if $a_{1}>a_{0}$ then the positive term is used and if $a_{1}<a_{0}$ then the negative term is used. Equation (31) can thus be used to determine the first phase duration for a given set of sail pitch and augmented clock angles. The resultant orbit inclination at the end of the first phase can be found by combining Eq. (26) and (28) to yield,

$$
\left\langle\frac{d i}{d t}\right\rangle= \pm \frac{2 \beta}{\pi} \frac{\sqrt{\mu}}{a^{3 / 2}}(\cos \alpha)^{2} \sin \alpha \sin \tilde{\gamma}
$$

where, $0 \leq \tilde{\gamma} \leq(\pi / 2)$ with the ' \pm ' term is used for a positive or negative rate of change of inclination. Using Eq. (31) and integrating yields,

$$
i_{1}=i_{0} \mp \frac{2 \tan \tilde{\gamma}}{3 \pi} \operatorname{Ln}\left(\frac{3 t \beta \sqrt{\mu} \mp a_{0}^{3 / 2} \csc \alpha(\sec \alpha)^{2} \sec \tilde{\gamma}}{\mp a_{0}^{3 / 2} \csc \alpha(\sec \alpha)^{2} \sec \tilde{\gamma}}\right)
$$

where the ' $\mp$ ' term is used for a positive or negative rate of change of semi-major axis, respectively. To find the time required for the second phase, to complete the required orbit plane change, Eq. (33) must be re-derived using the clock angle rather than the augmented clock angle to get,

$$
t_{2}=\mp \frac{a_{1}^{3 / 2}\left(i_{1}-i_{t}\right) \pi \csc \alpha(\sec \alpha)^{2}}{2 \beta \sqrt{\mu}}
$$

where, the ' $\mp$ ' term is used for positive or negative rate of change of inclination, $i_{t}$ is the target orbit inclination and the total orbit transfer duration is $t=\left(t_{1}+t_{2}\right)$.

\section{Hybrid Solar Sail, Constant Acceleration Electric Propulsion General Perturbations Approximation}

The analysis presented in the two proceeding sections can be repeated for a hybrid constant acceleration electric propulsion and solar sail propelled spacecraft. Once again assuming an initially, or nearly circular orbit the Lagrange-Gauss variational equations can be averaged, as seen in Eq. (25) \& (26). Recalling Eq. (27) \& (28), adopting the locally optimal control laws used previously and again using the augmented clock angle to define the solar sail out-of-plane thrust angle the averaged semi-major axis becomes,

$$
\left\langle\frac{\mathrm{d} a}{\mathrm{~d} t}\right\rangle= \pm \frac{a^{\frac{3}{2}}}{\pi \sqrt{\mu}} \int_{0}^{2 \pi}\left[\beta \frac{\mu}{a^{2}}(\cos \alpha)^{2} \sin \alpha \cos \tilde{\gamma}+\varepsilon_{0} \cos \tilde{\chi}\right](\theta) \mathrm{d} \theta
$$

where, $\chi$ is as previously defined in the 'Electric Propulsion' section, that is the electric propulsion out-of-plane thrust angle and where the ' \pm ' term is used for a positive or negative rate of change of semi-major axis. Defining,

$$
\begin{aligned}
& \Psi=\beta \sqrt{\mu}(\cos \alpha)^{2} \sin \alpha \cos \tilde{\gamma} \\
& \Xi=\frac{\varepsilon_{0} \cos \tilde{\chi}}{\sqrt{\mu}} \\
& \mathrm{N}_{0}=\frac{\sqrt{2 a_{0}} \Xi^{\frac{1}{4}}}{\Psi^{\frac{1}{4}}}
\end{aligned}
$$




$$
\begin{aligned}
& \mathrm{N}_{1}=\frac{\sqrt{2 a_{1}} \Xi^{\frac{1}{4}}}{\Psi^{\frac{1}{4}}} \\
& \Gamma_{0}=\frac{2 \sqrt{2 a_{0}} \Xi^{\frac{1}{4}} \Psi^{\frac{1}{4}}}{a_{0} \sqrt{\Xi}-\sqrt{2 a_{0}} \Xi^{\frac{1}{4}} \Psi^{\frac{1}{4}}+\sqrt{\Psi}} \\
& \Gamma_{1}=\frac{2 \sqrt{2 a_{1}} \Xi^{\frac{1}{4}} \Psi^{\frac{1}{4}}}{a_{1} \sqrt{\Xi}-\sqrt{2 a_{1}} \Xi^{\frac{1}{4}} \Psi^{\frac{1}{4}}+\sqrt{\Psi}} \\
& \Lambda=\frac{1}{2 \sqrt{2} \Xi^{\frac{3}{4}} \Psi^{\frac{1}{4}}}
\end{aligned}
$$

$$
\begin{gathered}
\mathrm{t}= \pm \frac{\Lambda}{2}\left\{2\left[\tan ^{-1}\left(1+\mathrm{N}_{1}\right)-\tan ^{-1}\left(1-\mathrm{N}_{1}\right)-\tan ^{-1}\left(1+\mathrm{N}_{0}\right)+\tan ^{-1}\left(1-\mathrm{N}_{0}\right)\right]\right. \\
\left.+\log \left(\left(1+\Gamma_{0}\right)\left(1-\Gamma_{1}\right)\right)\right\}
\end{gathered}
$$

where, again the ' \pm ' term is used for a positive or negative rate of change of semi-major axis, respectively. That is, if $a_{1}>a_{0}$ then the positive term is used and if $a_{1}<a_{0}$ then the negative term is used. However, Eq. (43) cannot be algebraically re-arranged to give the semi-major axis as a function of time, meaning that the inclination as a function of time cannot be algebraically determined for a varying semi-major axis.

The time taken to alter the inclination between two values can be determined for a constant radius by using Eq. (26) \& (28) and adopting the locally optimal inclination control law where $\chi=\pi / 2$ and $3 \pi / 2$ and $\delta=0$ and $\pi$, switching at true anomaly $\pi / 2$ and $3 \pi / 2$. Eq. (26) can thus be solved to give the inclination as a function of time as,

$$
i(t)=i_{0} \pm \frac{2}{\pi}\left(\beta \frac{\sqrt{\mu}}{a^{3 / 2}}(\cos \alpha)^{2} \sin \alpha+\varepsilon_{0} \sqrt{\frac{a}{\mu}}\right) t
$$

and hence the time between two inclinations is,

$$
t= \pm \frac{\pi}{2} \frac{\left(i_{1}-i_{0}\right)}{\left(\beta \frac{\sqrt{\mu}}{a^{3 / 2}}(\cos \alpha)^{2} \sin \alpha+\varepsilon_{0} \sqrt{\frac{a}{\mu}}\right)}
$$

It is noted that Eq. (45) can be simplified towards Eq. (15) or (34) when $\varepsilon_{0} \rightarrow 0$ or $\beta \rightarrow 0$, respectively.

\section{Hybrid Solar Sail, Constant Thrust Electric Propulsion General Perturbations Approximation}

As before, the analysis presented in the proceeding sections can be repeated for a hybrid constant thrust electric propulsion and solar sail propelled spacecraft. Once again assuming an initially, or nearly circular orbit the Lagrange-Gauss variational equations can be averaged, as seen in Eq. (25) \& (26). Recalling Eq. (27) \& (28) it is noted that the sail lightness number will be a function of time, as the spacecraft mass reduces due to the use of electric propulsion system propellant. From Eq. (8), the spacecraft mass can be written as,

$$
m(t)=\frac{\left|\mathbf{T}_{c}\right|}{\varepsilon_{0}}\left(1-\frac{\varepsilon_{0}}{c} t\right)
$$

where, $\mathbf{T}_{c}$ is the constant thrust of the electric propulsion system. Noting that the sail lightness number can be written as $\beta=\left(\sigma^{*} / \sigma\right)$, where $\sigma^{*}=1.53 \mathrm{~g} / \mathrm{m}^{2}$ is the critical sail loading, ${ }^{6}$ and that the sail loading can be written as $\sigma=\left(m(t) / A_{\text {sail }}\right)$, where $A_{\text {sail }}$ is the surface area of the solar sail, the sail lightness number can be expressed as,

$$
\beta=\frac{A_{\text {sail }} \varepsilon_{0} \sigma^{*}}{\left|\mathbf{T}_{c}\right|\left(1-\frac{\varepsilon_{0}}{C} t\right)}
$$

and Eq. (27) \& (28) become,

$$
\begin{aligned}
& T=\frac{A_{\text {sail }} \varepsilon_{0} \sigma^{*}}{\left|\mathbf{T}_{c}\right|\left(1-\frac{\varepsilon_{0}}{C} t\right)} \frac{\mu}{a^{2}} \cos \alpha^{2} \sin \alpha \sin \delta \\
& N=\frac{A_{\text {sail }} \varepsilon_{0} \sigma^{*}}{\left|\mathbf{T}_{c}\right|\left(1-\frac{\varepsilon_{0}}{C} t\right)} \frac{\mu}{a^{2}} \cos \alpha^{2} \sin \alpha \cos \delta
\end{aligned}
$$


Adopting the locally optimal control laws used previously and again using the augmented clock angle to define the solar sail out-of-plane thrust angle the averaged semi-major axis becomes,

$$
\left\langle\frac{\mathrm{d} a}{\mathrm{~d} t}\right\rangle= \pm \frac{a^{\frac{3}{2}}}{\pi \sqrt{\mu}} \int_{0}^{2 \pi}\left[\frac{A_{\text {sail }} \varepsilon_{0} \sigma^{*}}{\left|\mathbf{T}_{c}\right|\left(1-\frac{\varepsilon_{0}}{C} t\right)} \frac{\mu}{a^{2}}(\cos \alpha)^{2} \sin \alpha \cos \tilde{\chi}+\frac{\varepsilon_{0}}{\left(1-\frac{\varepsilon_{0}}{c} t\right)} \cos \tilde{\chi}\right](\theta) \mathrm{d} \theta
$$

where, $\chi$ is as previously defined in the 'Electric Propulsion' section, that is the electric propulsion out-of-plane thrust angle and where the ' \pm ' term is used for a positive or negative rate of change of semi-major axis.

Re-defining Eq. (36) and (37) as,

$$
\begin{aligned}
& \Psi=\frac{A_{\text {sail }} \sigma^{*}}{\left|\mathbf{T}_{c}\right|} \sqrt{\mu}(\cos \alpha)^{2} \sin \alpha \cos \tilde{\chi} \\
& \Xi=\frac{\cos \tilde{\chi}}{\sqrt{\mu}}
\end{aligned}
$$

and recalling Eq. (38) - (42), direct integration yields,

$$
t=\frac{c}{\varepsilon_{0}}\left[1-e^{\left[\frac{\Lambda}{c}\left( \pm \tan ^{-1}\left(1-\mathrm{N}_{0}\right) \mp \tan ^{-1}\left(1+\mathrm{N}_{0}\right) \mp \tan ^{-1}\left(1-\mathrm{N}_{1}\right) \pm \tan ^{-1}\left(1+\mathrm{N}_{1}\right)\right)\right]}\left(1+\Gamma_{0}\right)^{ \pm \frac{\Lambda}{2 c}}\left(1-\Gamma_{0}\right)^{ \pm \frac{\Lambda}{2 c}}\right]
$$

where, the ' $\mp$ ' and ' \pm ' are used for positive/negative rates of change of semi-major axis. As with the constant acceleration hybrid, Eq. (53) cannot be algebraically re-arranged to give the semi-major axis as a function of time, meaning that the inclination as a function of time cannot be algebraically determined for a varying semi-major axis. The time taken to alter the inclination between two values can be determined for a constant radius by using Eq. (26) and (48) and adopting the locally optimal inclination control law where $\chi=\pi / 2$ and $3 \pi / 2$ and $\delta=0$ and $\pi$, switching at true anomaly $(\pi / 2)$ and $(3 \pi / 2)$. Eq. (26) can thus be solved to give the inclination as a function of time as,

$$
i_{1}=i_{0} \mp \frac{2}{\pi} c \log \left(1-\frac{t \varepsilon_{0}}{c}\right)\left(\frac{A_{\text {sail }} \sigma^{*}}{\left|\mathbf{T}_{c}\right|} \frac{\sqrt{\mu}}{a^{3 / 2}}(\cos \alpha)^{2} \sin \alpha+\sqrt{\frac{a}{\mu}}\right)
$$

and hence the time between two inclinations is,

$$
t=\mp \frac{c}{\varepsilon_{0}}\left(-1+e^{\frac{a^{3 / 2} \pi\left( \pm i_{0} \mp i_{1}\right) T_{h}}{2 c\left(a^{3 / 2}\left|\mathbf{T}_{c}\right| \sqrt{\frac{a}{\mu}}+A_{\text {sail }} \sqrt{\mu} \sigma^{*}(\cos \alpha)^{2} \sin \alpha\right)}}\right)
$$

It is noted that Eq. (55) can be simplified towards Eq. (21) and (34) when $\varepsilon_{0} \rightarrow 0$ or $\beta \rightarrow 0$, respectively.

\section{Analysis of Electric Propulsion Solar Polar Transfer}

\section{A. Constant Acceleration}

Considering a constant acceleration low-thrust propelled spacecraft transfer from a circular 1 au orbit within the ecliptic plane to a circular orbit of semi-major axis $a_{1}$ inclined at a target inclination of $i_{t}$, it is found that the total transfer time (in seconds) can be written in the form,

$$
t=\frac{1}{\varepsilon_{0}} \frac{1}{\sqrt{a_{1}}}\left[\mathfrak{A} i_{t}+\frac{\mathfrak{B}-\mathfrak{C} \sqrt{a_{1}}}{\cos \chi}+\tan \chi\left(\mathfrak{D} \log \left[a_{1}\right]-\mathfrak{E}\right)\right]
$$

where, $\mathfrak{A}, \mathfrak{B}, \mathfrak{C}, \mathfrak{D}$ and $\mathfrak{E}$ are all constants determined by the orbit parameters, the value of which is not relevant to this analysis. From Eq. (56) it is seen that as the out-of-plane thrust angle tends to 90 degrees the trip time is minimized. However, it should be noted that Eq. (56) is not valid when $\chi=(\pi / 2)$. Using Eq. (9) \& (10), Eq. (56) can be re-formulated for the case $\chi=(\pi / 2)$, in other words, when the inclination is changed at 1 au and then the semi-major axis changed only when the target inclination has been reached. As such, Eq. (56) can be written in the form,

$$
t=\frac{1}{\varepsilon_{0}}\left[\mathfrak{A} i_{t}+\frac{\mathfrak{B}}{\sqrt{a_{1}}}-\mathfrak{C}\right]
$$

where, $\mathfrak{A}, \mathfrak{B}$ and $\mathfrak{C}$ are constants, but are not equal in value to those in Eq. (56).

For a constant acceleration of $0.2 \mathrm{~mm} \mathrm{~s}^{-2}$, equivalent to a thrust of $200 \mathrm{mN}$ on a $1000 \mathrm{~kg}$ spacecraft, it is found that the transfer to a 82.75 degree, circular orbit at 0.48 au (a solar polar orbit in a 1:3 Earth resonant orbit) would require approximately 17.5 years to complete (with first phase of 2.09 years) if the semi-major axis changed in phase 1 , i.e. $\tilde{\chi}=0$. However, this trip time can be reduced to 12.8 years by changing the inclination at 1 au rather than at the target orbit radius, i.e. $\tilde{\chi}=(\pi / 2)$. The saving in trip time is from the inclination change phase, with the 
semi-major axis phase remaining of the same duration. It is found that the same general trends are observed when the target inclination is reduced.

\section{B. Constant Thrust}

Considering a constant thrust low-thrust propelled spacecraft transfer from a circular 1 au orbit within the ecliptic plane to a circular orbit of semi-major axis $a_{1}$ inclined at a target inclination of $i_{t}$, it is found that the total transfer time (in seconds) can be written in the form,

$$
t=\frac{\mathrm{c}}{\varepsilon_{0}}\left[2-e^{\frac{\mathfrak{A} \sqrt{a_{1}}-\mathfrak{B}}{c \sqrt{a_{1}} \cos \chi}}-e^{\frac{1}{c \sqrt{a_{1}}}\left[\tan \chi\left(\mathcal{D}-\mathfrak{B} \log \left[\mathfrak{A}-\left(\frac{\mathfrak{A} \sqrt{a_{1}}-\mathfrak{B}}{\sqrt{a_{1}}}\right)\right]\right)-\mathfrak{C i}_{t}\right]}\right]
$$

where, $\mathfrak{A}, \mathfrak{B}, \mathfrak{C}$, and $\mathfrak{D}$ are all constants determined by the orbit parameters, the value of which is not relevant to this analysis, and $c$ is the propulsion system exhaust velocity. Equation (58) is visualized in Fig. 3 for an initial acceleration, $\varepsilon_{0}=0.2 \mathrm{~mm} / \mathrm{s}^{2}$, say, a fixed thrust of $200 \mathrm{mN}$ on a $1000 \mathrm{~kg}$ spacecraft, and $i_{t}=82.75$ degrees, where it is seen that the trip time is minimized when $\tilde{\chi}=0$.

For an initial acceleration of $0.2 \mathrm{~mm} \mathrm{~s}^{-2}$, it is found that the minimum transfer time, i.e. using $\tilde{\chi}=0$, to a 82.75 degree, circular orbit at 0.48 au is approximately 8.63 years (with first phase of 1.84 years). However, from Eq. (8) it is noted that this transfer requires a fuel mass fraction of approximately $109 \%$; assuming a propulsion system exhaust velocity, $c$, of $50000 \mathrm{~m} / \mathrm{s}$, equivalent to a specific impulse of approximately $5097 \mathrm{~m} / \mathrm{s}$. It is found that the same general trends are observed when the target inclination is reduced. For example, the trip time is reduced to 7.48 years (with first phase of 1.84 years) for $i_{t}=52.75$ degrees, peak solar latitude of 60 degrees, with the fuel mass fraction reduced to $94.4 \%$; assuming the same propulsion system exhaust velocity as before.

\section{Analysis of Solar Sail Propulsion Solar Polar Transfer}

Considering a solar sail propelled spacecraft transfer from a circular 1 au orbit within the ecliptic plane to a circular orbit of semi-major axis $a_{1}$ inclined at a target inclination of $i_{t}$, it is found that the total transfer time (in seconds) can be written in the form,

$$
t=\frac{1}{\pi \beta}\left[\frac{1}{\cos \gamma}\left(\mathfrak{A}-\mathfrak{B} a_{1}^{\frac{3}{2}}\right)+a_{1}^{\frac{3}{2}}\left(\mathfrak{C i}_{t}+\tan \gamma\left(\mathfrak{B} \log \left[a_{1}\right]-\mathfrak{D}\right)\right)\right]
$$

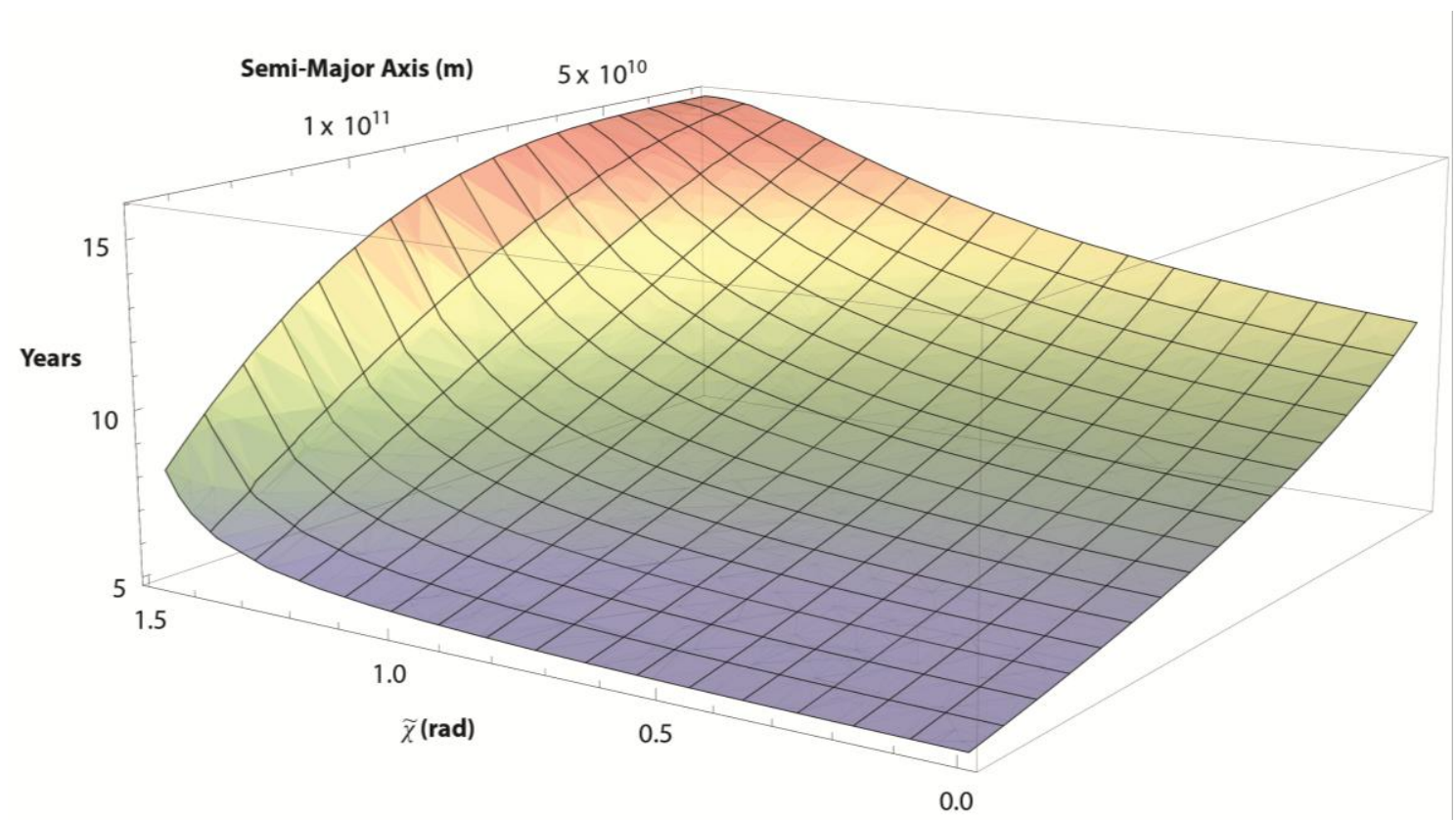

Fig. 3 Time to a solar polar orbit using a $200 \mathrm{mN}$ constant thrust electric propulsion system on a $1000 \mathrm{~kg}$ spacecraft to target semi-major axis between 0.2 au and 0.9 au 
where, $\mathfrak{A}, \mathfrak{B}, \mathfrak{C}$ and $\mathfrak{D}$ are all constants determined by the orbit parameters, the value of which is not relevant to this analysis. Equation (59) is visualized in Fig. 4 for a solar sail with a characteristic acceleration of $0.2 \mathrm{~mm} / \mathrm{s}^{2}$ (lightness number, $\beta=0.03372$ ) and $i_{t}=82.75$ degrees. It is seen from Eq. (59) and Fig. 4 that the trip time is minimized when the out-of-plane thrust angle is $0<\gamma<(\pi / 2)$ and that a turning point can be identified when the trip time is minimized. It is found that the same general trends are observed when the target inclination is reduced.

Table 1 details the optimal general perturbations solutions for transfers from a circular 1 au orbit within the ecliptic plane to an 82.75 degree and 52.75 degree circular orbit (peak solar latitudes of 90 and 60 degrees, respectively) at a range of target orbit radius. It is seen that, independent of the target inclination, the inclination at the end of the first phase is typically between $7-9$ degrees, whilst the out-of-plane angle in phase $1, \tilde{\gamma}$, decreases as the target radius is decreased. It is also noted that a turning point occurs in the end of phase 1 inclination as the orbit radius is reduced to very low values.

From Table 1 a solar sail with a characteristic acceleration of $0.5 \mathrm{~mm} / \mathrm{s}^{2}$ (lightness number, $\beta=0.0843$ ) will reach a 82.75 degree orbit at a radius of 0.63 au, 0.48 au and 0.4 au in 6.2 years, 4.6 years and 3.9 years, respectively. Similarly, a solar sail with a characteristic acceleration of $0.1 \mathrm{~mm} / \mathrm{s}^{2}$ (lightness number, $\beta=0.0169$ ) will reach a 82.75 deg. orbit at a radius of 0.63 au, 0.48 au and 0.4 au in 30.8 years, 23.1 years and 19.5 years, respectively.
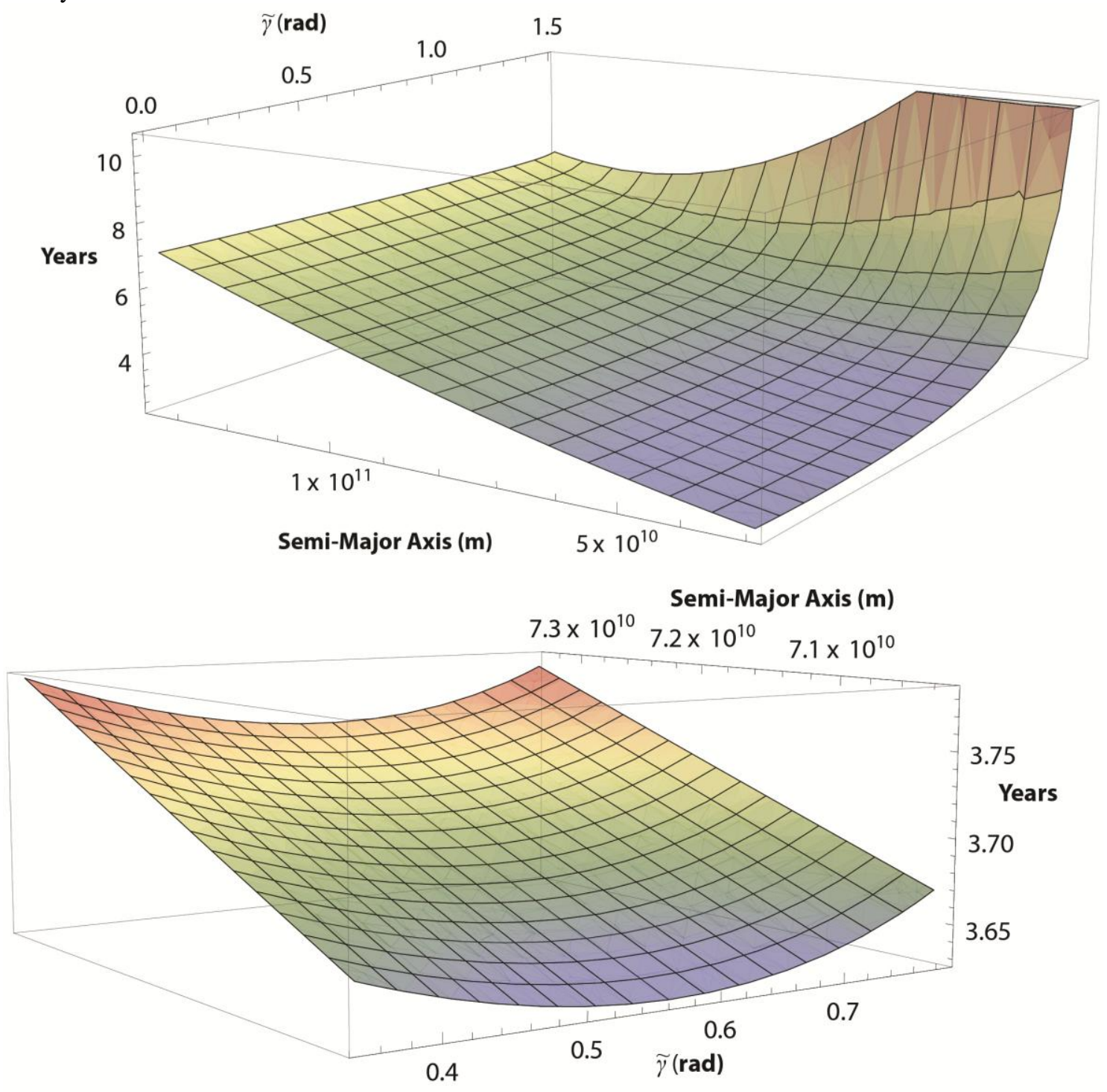

Fig. 4 Time to a solar polar orbit using a solar sail with characteristic acceleration $0.2 \mathrm{~mm} / \mathrm{s}^{2}$ to target semi-major axis between 0.2 au and 0.9 au (top) and 0.47 au and 0.49 au (bottom) 
Table 1 Optimal general perturbations solutions for solar sail orbit transfer to solar polar orbit at 82.75 degrees inclination and 52.75 degrees inclination; peak solar latitudes of 90 and 60 degrees, respectively.

\begin{tabular}{|c|c|c|c|c|c|}
\hline $\begin{array}{c}\text { Target } \\
\text { Radius } \\
\text { (au) }\end{array}$ & $\begin{array}{c}\text { Out-of-plane angle } \\
\text { in phase one, } \widetilde{\gamma} \\
\text { (degrees) }\end{array}$ & $\begin{array}{c}\text { Inclination at } \\
\text { end of phase 1 } \\
\text { (degrees) }\end{array}$ & $\begin{array}{c}\text { Phase 1 } \\
\text { duration } \\
\text { (years) }\end{array}$ & $\begin{array}{c}\text { Total Duration } \\
\text { to 82.75 } \\
\text { (years) }\end{array}$ & $\begin{array}{c}\text { Total Duration } \\
\text { to 52.75 } \\
\text { (years) }\end{array}$ \\
\hline 0.90 & 67.4 & 4.61 & $\frac{0.052384}{\beta}$ & $\frac{0.808725}{\beta}$ & $\frac{0.518342}{\beta}$ \\
\hline 0.75 & 53.1 & 6.99 & $\frac{0.0804637}{\beta}$ & $\frac{0.638326}{\beta}$ & $\frac{0.417424}{\beta}$ \\
\hline 0.63 & 43.9 & 8.10 & $\frac{0.0956106}{\beta}$ & $\frac{0.51877}{\beta}$ & $\frac{0.348703}{\beta}$ \\
\hline 0.55 & 38.2 & 8.57 & $\frac{0.103787}{\beta}$ & $\frac{0.446827}{\beta}$ & $\frac{0.308103}{\beta}$ \\
\hline 0.48 & 33.3 & 8.78 & $\frac{0.110031}{\beta}$ & $\frac{0.388895}{\beta}$ & $\frac{0.275793}{\beta}$ \\
\hline 0.40 & 27.7 & 8.79 & $\frac{0.116338}{\beta}$ & $\frac{0.328459}{\beta}$ & $\frac{0.242419}{\beta}$ \\
\hline 0.30 & 20.8 & 8.34 & $\frac{0.123219}{\beta}$ & $\frac{0.261829}{\beta}$ & $\frac{0.205945}{\beta}$ \\
\hline
\end{tabular}

From Table 1 it is noted that the total transfer time reduces as the target orbit radius is reduced. This can be understood by considering the ratio of Phase 1 duration, to the total mission duration. It is seen that the majority of transfer time is in Phase 2, when the target orbit radius has been achieved and due to the $\left(1 / r^{2}\right)$ variation in sail performance, the sail is at peak performance level. Whilst this result cannot be generalized for the optimal case due to the variation in the optimal out of plane thrust angle, $\tilde{\gamma}$, with target orbit radius, the optimal out of plane thrust angle can be approximated as a third order polynomial, giving,

$\tilde{\gamma} \cong 1.1525 a_{1}^{3}-1.6019 a_{1}^{2}+1.9291 a_{1}-0.1034$

where, $a_{1}$ is measured in au. Note that Eq. (60) is valid for a target inclination greater than the inclination at the end of phase 1, approximately 9 degrees. Note further that Eq. (60) has a co-efficient of determination, $\mathrm{R}^{2}$, value, based on the values in Table 1 of 0.99998.

Using Eq. (60), Eq. (59) can be re-written as,

$$
\begin{aligned}
& t=\frac{1}{\pi \beta}\left[\frac{\left(\mathfrak{A}+\mathfrak{B} a_{1}^{\frac{3}{2}}\right)}{\cos \left[\mathfrak{C}\left(\mathfrak{D}+a_{1}\right)\left(\mathfrak{E}+\mathfrak{F} a_{1}+a_{1}^{2}\right)\right]}\right. \\
& \left.+a_{1}^{\frac{3}{2}}\left(\mathfrak{5} i_{t}+\left(\mathfrak{H}+\mathfrak{J} \log \left[a_{1}^{\frac{3}{2}}\right]\right) \tan \left[\mathfrak{I}+\mathfrak{K} a_{1}+\mathfrak{L} a_{1}^{2}+\mathfrak{M} a_{1}^{3}\right]\right)\right]
\end{aligned}
$$

where, $a_{1}$ is measured in metres again and not au as in Eq. (60), $\mathfrak{A}, \mathfrak{B}, \mathfrak{C}, \mathfrak{D}, \mathfrak{E}, \mathfrak{F}, \mathfrak{W}, \mathfrak{H}, \mathfrak{I}, \mathfrak{J}, \mathfrak{K}, \mathfrak{L}$ and $\mathfrak{M}$ are constants determined by the orbit parameters, distinct from those used in Eq. (59) and defined in Table 2. 
Table 2 Constants for Eq. (61)

\begin{tabular}{|c|l|}
\hline \multicolumn{1}{|c|}{ Constant } & \multicolumn{1}{|c|}{ Value } \\
\hline $\mathfrak{A}$ & 0.43303013661188056 \\
\hline $\mathfrak{B}$ & $-7.483934949353608 \times 10^{-18}$ \\
\hline $\mathfrak{C}$ & $3.4425263133185114 \times 10^{-34}$ \\
\hline $\mathfrak{D}$ & $-8.392144253966071 \times 10^{+9}$ \\
\hline $\mathfrak{E}$ & $3.5784751639989546 \times 10^{+22}$ \\
\hline $\mathfrak{S}$ & $-1.995309183700735 \times 10^{+11}$ \\
\hline $\mathfrak{S}$ & $3.5267212585249795 \times 10^{-17}$ \\
\hline $\mathfrak{T}$ & $2.8885612757886467 \times 10^{-16}$ \\
\hline $\mathfrak{I}$ & $-7.483934949353608 \times 10^{-18}$ \\
\hline $\mathfrak{I}$ & 0.103382782379 \\
\hline $\mathfrak{K}$ & $-1.2895443276860758 \times 10^{-11}$ \\
\hline $\mathfrak{L}$ & $7.157806142290288 \times 10^{-23}$ \\
\hline $\mathfrak{M}$ & $-3.4425263133185114 \times 10^{-34}$ \\
\hline
\end{tabular}

\section{A. Validation}

In [2] a typical two-phase transfer trajectory to an orbit radius of 0.48 au, at 90 degrees to the ecliptic plane is derived using a Calculus of Variations approach for a solar sail with a characteristic acceleration of $0.5 \mathrm{~mm} / \mathrm{s}^{2}$. The first phase is 578 days and increases the orbit inclination to approximately 15 degrees, whilst reducing the orbit radius to $0.48 \mathrm{au}$, whilst the second phase is 1212 days long and increases the orbit inclination to 90 degrees; giving a total transfer duration of 1790 days, or 4.90 years. It should be noted that the inclination at the end of phase 1 was selected based on empirical evidence as being the quasi-optimal target inclination at the end of phase 1. In [10] a semi-analytical method, which requires the use of numerical methods, is compared against the results presented in [2]. The method developed in [10] remains unable to derive the optimal end of phase 1 orbit inclination; however, once again using an a priori selection of 15 degrees to divide the transfer into two separately considered phases is able to estimate a total transfer duration of 1799 days, or 4.93 years. Furthermore, [10] derives an optimal orbit inclination at the end of phase 1 as approximately 11 degrees, reducing the trip time to 1787 days, or 4.89 years.

Using Eq. (61) the total trip time to an orbit radius of 0.48 au, at 90 degrees to the ecliptic plane, for a solar sail with a characteristic acceleration of $0.5 \mathrm{~mm} / \mathrm{s}^{2}$, is found to be 4.94 years, with a derived ideal orbit inclination of 8.8 degrees at the end of phase 1, as expected from Table 1. It is noted that the minimum transfer time using Eq. (61) is approximately $0.8 \%$ more than the optimal transfer time derived in [2] and approximately $1 \%$ more than the optimal transfer time derived in [10]. The effect of varying the orbit inclination at the end of phase 1 is fully and easily quantified in Fig. 5.

\section{B. Attainable Orbit Space}

Using Eq. (61) the attainable orbit space within five years is seen in Fig. 6, assuming an initially circular orbit at 1 au within the Ecliptic plane and a two-phase transfer. It is seen in Fig. 6 that with a sail characteristic acceleration of $0.2 \mathrm{~mm} / \mathrm{s}^{2}$ a peak solar latitude of 60 degrees cannot be reached in 5-years, whilst with a sail characteristic acceleration of 0.3 and $0.4 \mathrm{~mm} / \mathrm{s}^{2}$ a peak solar latitude of 60 degrees at orbit radius 0.43 and 0.62 au, respectively, can be reached in a similar timeframe. It is also seen in Fig. 6 that restricting sail characteristic acceleration to below $0.5 \mathrm{~mm} / \mathrm{s}^{2}$, it is not possible using a two-phase 5-year transfer trajectory to attain a solar orbit that maintains the observer-to-solar pole zenith angle, OZA, (see Fig. 7) below 30 degrees for 25 days; the approximate sidereal rotation period of the Sun, based on a solar latitude of 16 degrees. It is seen however that a sail characteristic acceleration below $0.5 \mathrm{~mm} / \mathrm{s}^{2}$ can, using a two-phase 5 -year transfer trajectory, attain a solar orbit that maintains the OZA below 40 degrees for 25 days.

From Fig. 6 it is apparent that a range of two-phase transfer trajectories are possible within any set of mission requirements. Assuming the spacecraft is required to reach an orbit inclination of 52.75 degrees in 5-years; equivalent to a peak solar latitude of 60 degrees, and that the final OZA should be less than 40 degrees, and ideally should be less than 30 degrees, during a sidereal rotation period of the Sun, the range of two-phase transfer trajectories are defined by the OZA 30 and 40 degree contour lines, giving a target solar radii between 0.550 au and 0.447 au. Table 3 summaries a range of possible mission architectures, with an increasing level of technology requirement on the solar sail, ranging from a characteristic acceleration of $0.28 \mathrm{~mm} \mathrm{~s}^{-2}$ up to $0.54 \mathrm{~mm} \mathrm{~s}^{-2}$. 


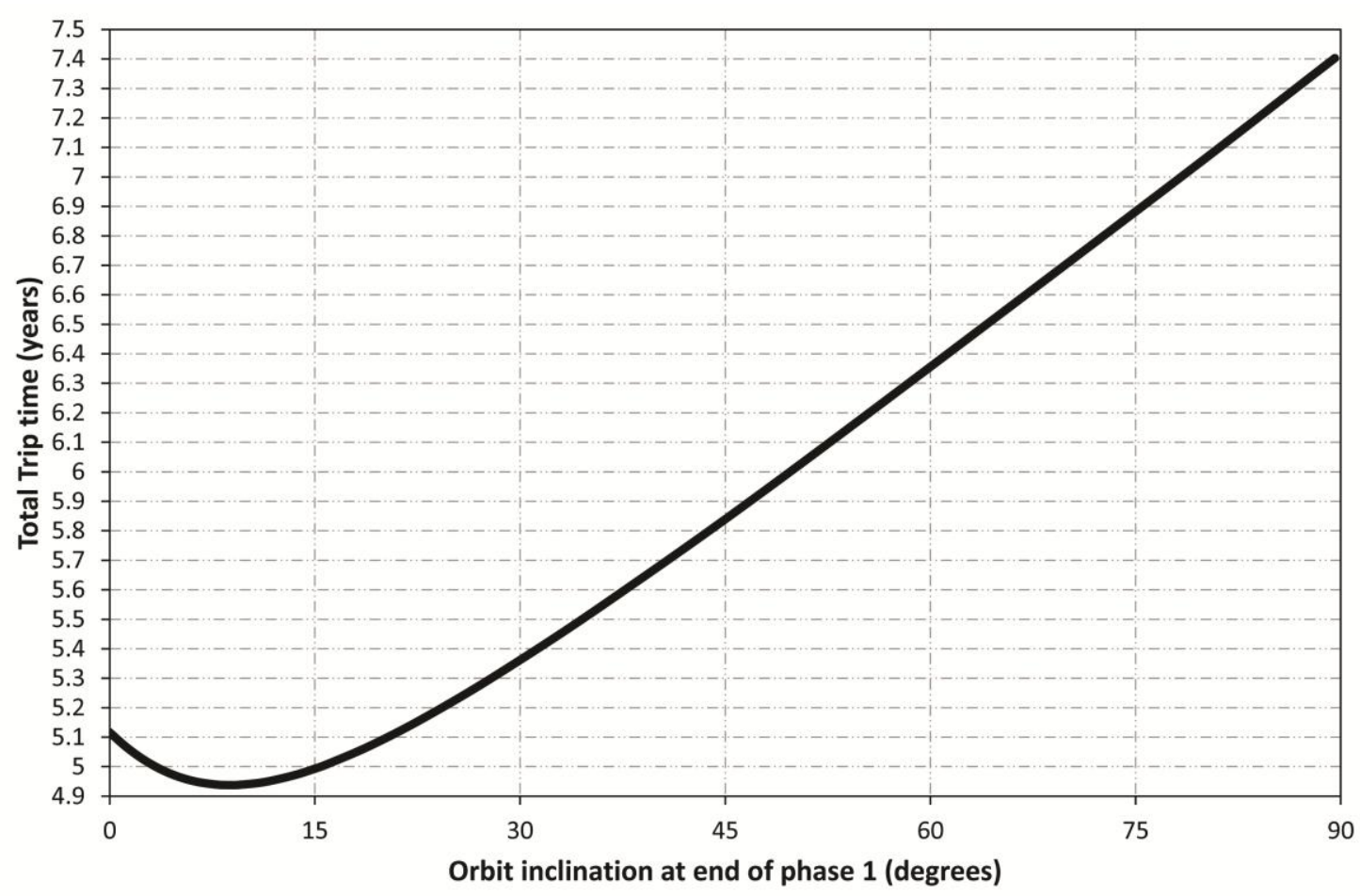

Fig. 5 The effect of varying the orbit inclination at the end of phase 1 on time from a circular 1 au orbit in the ecliptic plane to a 0.48 au orbit with 90 degrees inclination.

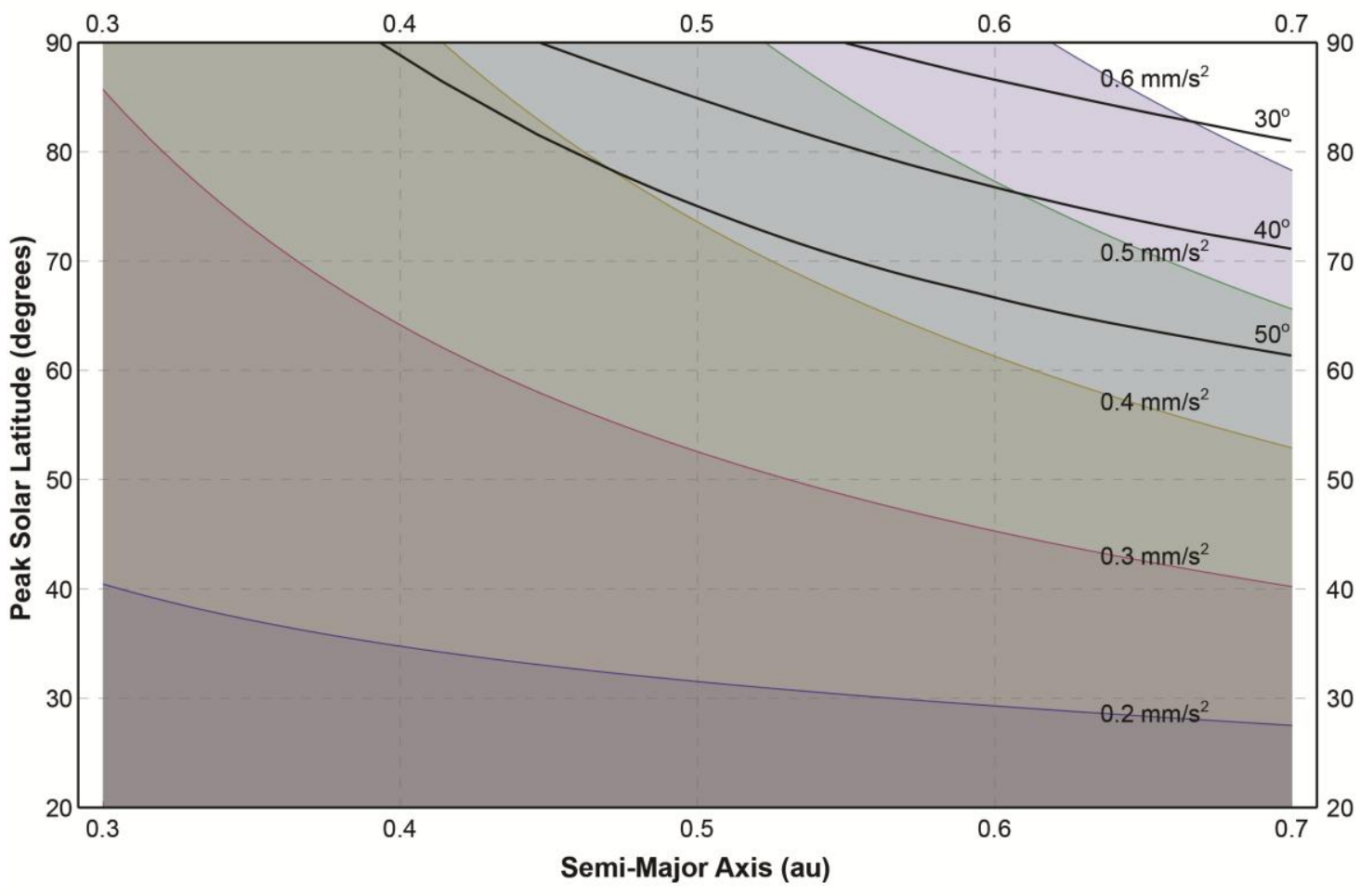

Fig. 6 Attainable peak solar latitude and semi-major axis within 5-years for a sail characteristic accelerations of $0.2 \mathrm{~mm} \mathrm{~s}^{-2}$ (bottom); $0.3 \mathrm{~mm} \mathrm{~s}^{-2} ; 0.4 \mathrm{~mm} \mathrm{~s}^{-2} ; 0.5 \mathrm{~mm} \mathrm{~s}^{-2} ; 0.6 \mathrm{~mm} \mathrm{~s}^{-2}$ (top); 25-day maximum observer-to-solar pole zenith angle contours also shown

American Institute of Aeronautics and Astronautics 


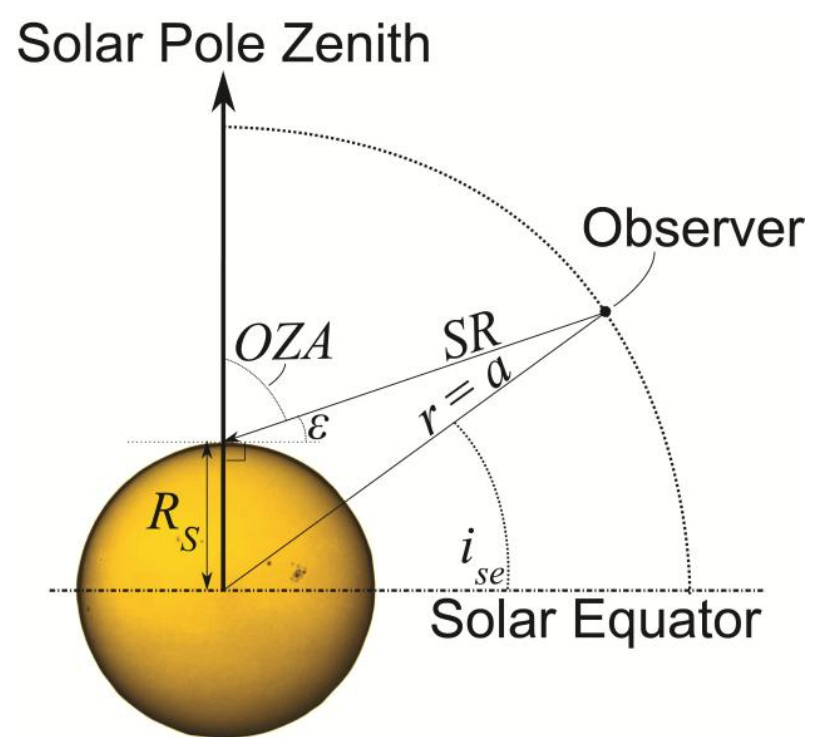

Fig. 7 Solar pole visibility; note that the solar equator is offset from the ecliptic plane by 7.25 degrees

Table 3 Summary and comparison of solar sail mission architecture options and requirements; superscript number indicates number of trajectory phases.

\begin{tabular}{|l|l|l|l|l|l|l|}
\cline { 2 - 7 } \multicolumn{1}{c|}{} & \multicolumn{1}{c|}{$\mathbf{A}^{2}$} & \multicolumn{1}{c|}{$\mathbf{A +}^{2}$} & \multicolumn{1}{c|}{$\mathbf{B}^{2}$} & \multicolumn{1}{c|}{$\mathbf{B}^{2}$} & \multicolumn{1}{c|}{$\mathbf{C}^{2}$} & \multicolumn{1}{c|}{$\mathbf{+}^{2}$} \\
\hline $\begin{array}{l}\text { Solar pole maximum } \\
\text { OZA in one-sidereal } \\
\text { rotation period }\end{array}$ & $50 \mathrm{deg}$. & $50 \mathrm{deg}$. & $40 \mathrm{deg}$. & $40 \mathrm{deg}$. & $30 \mathrm{deg}$. & $30 \mathrm{deg}$. \\
\hline Target orbit radius & $0.397 \mathrm{au}$ & $0.397 \mathrm{au}$ & $0.461 \mathrm{au}$ & $0.461 \mathrm{au}$ & $0.559 \mathrm{au}$ & $0.559 \mathrm{au}$ \\
\hline $\begin{array}{l}\text { Required sail } \\
\text { characteristic } \\
\text { acceleration }\end{array}$ & $0.29 \mathrm{~mm} / \mathrm{s}^{2}$ & $0.39 \mathrm{~mm} / \mathrm{s}^{2}$ & $0.32 \mathrm{~mm} / \mathrm{s}^{2}$ & $0.44 \mathrm{~mm} / \mathrm{s}^{2}$ & $0.37 \mathrm{~mm} / \mathrm{s}^{2}$ & $0.54 \mathrm{~mm} / \mathrm{s}^{2}$ \\
\hline Phase 1 duration & 2.4 years & 1.8 years & 2.1 years & 1.5 years & 1.7 years & 1.1 years \\
\hline $\begin{array}{l}\text { Inclination at end of } \\
\text { phase } 1\end{array}$ & $8.8^{\circ}$ & $8.8^{\circ}$ & $8.8^{\circ}$ & $8.8^{\circ}$ & $8.5^{\circ}$ & $8.5^{\circ}$ \\
\hline Time to $60^{\circ}$ latitude & 5.0 years & 3.7 years & 5.0 years & 3.6 years & 5.0 years & 3.4 years \\
\hline Time to $90^{\circ}$ latitude & 6.8 years & 5.0 years & 7.0 years & 5.0 years & 7.3 years & 5.0 years \\
\hline $\begin{array}{l}\text { Time to } 50^{\circ} \mathrm{OZA} \\
\text { view pole over a } \\
\text { sidereal rotation }\end{array}$ & 6.8 years & 5.0 years & 6.3 years & 4.5 years & 5.8 years & 4.0 years \\
\hline $\begin{array}{l}\text { Time to } 40^{\circ} \mathrm{OZA} \\
\text { view pole over a } \\
\text { sidereal rotation }\end{array}$ & $\mathrm{n} / \mathrm{a}$ & $\mathrm{n} / \mathrm{a}$ & 7.0 years & 5.0 years & 6.5 years & 4.5 years \\
\hline $\begin{array}{l}\text { Time to } 30^{\circ} \mathrm{OZA} \\
\text { view pole over a } \\
\text { sidereal rotation }\end{array}$ & $\mathrm{n} / \mathrm{a}$ & $\mathrm{n} / \mathrm{a}$ & $\mathrm{n} / \mathrm{a}$ & $\mathrm{n} / \mathrm{a}$ & 7.3 years & 5.0 years \\
\hline
\end{tabular}

\section{Strawman System Mass Budget}

A generic gossamer structure / solar sail model is used to allow general top-level technology capabilities to be analyzed and quantified. Noting that the possible film substrates typically considered in gossamer structures range in density from Polyethylene terephthalate (PET) at $1350 \mathrm{~kg} / \mathrm{m}^{3}$ to LaRCT-CP1 at $1430 \mathrm{~kg} / \mathrm{m}^{3}$, the generic model is based on Kapton film as this is one of the heaviest films likely to be used $\left(1420 \mathrm{~kg} / \mathrm{m}^{3}\right)$. In addition to the film, an allocation of $0.42 \mathrm{~g} / \mathrm{m}^{2}$ is given for adhesives and edge enhancement, but without swivels and without constant force springs. Sail film thickness can be arbitrarily selected; however, $7.5 \mu \mathrm{m}$ and $2.5 \mu \mathrm{m}$ films will be considered. 
Assuming a 3-axis stabilized gossamer structure, as opposed to a spin-stabilized structure, the support booms for the structure are a primary technology requirement. The impact of boom technology can hence also be quantified within the generic gossamer structure / solar sail model as shown in Fig. 8. Note within Fig. 8 the DLR boom technology is described within [21 \& 22], whilst the CoilAble booms are described in [23]. It should also be noted that the inflatable booms developed by L' Garde ${ }^{24-26}$ for use in the NASA-funded Sunjammer mission have a lower specific mass than the booms considered in Fig. 8; however, this data is not in the public domain.

Using the generic gossamer structure model in Fig. 8, a platform mass allocation can be determined for a given gossamer surface area, and from this, the approximate science suite payload mass allocation can be estimated as around $20 \%$ of the platform mass. ${ }^{27}$ Platform and science suite payload mass allocation budgets are shown in Table 4 - Table 9 for the mission architectures shown in Table 3, where it is seen that depending on the sail size and mission architecture a science suite payload mass allocation of up to $60 \mathrm{~kg}$ can be reasonably anticipated.

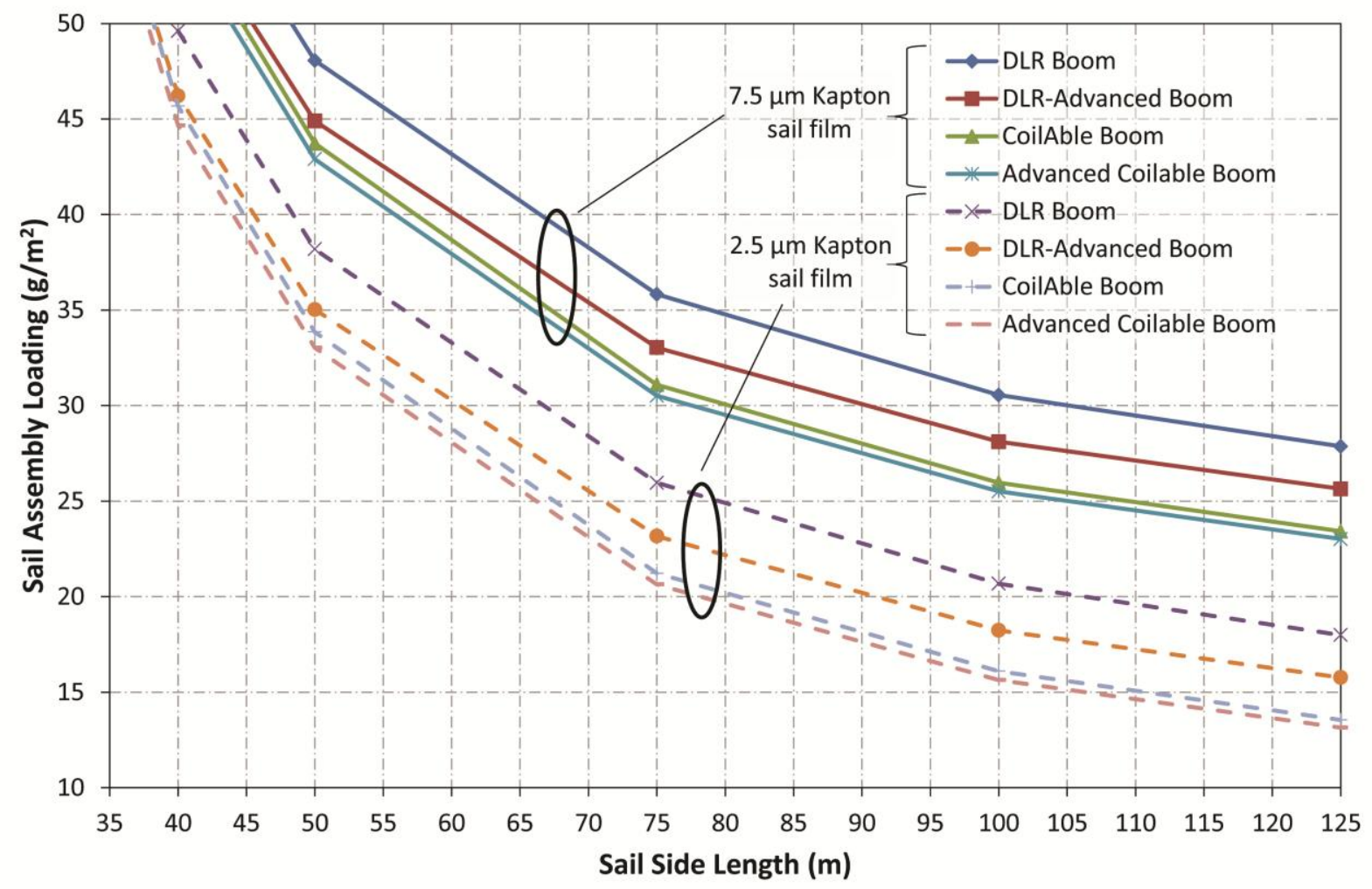

Fig. 8 Sail assembly loading using generic gossamer structure / solar sail model over a range of sail lengths and technology levels.

Table 4 Summary of mission architecture option $\mathbf{A}^{2}$ mass allowance estimates, including ROM payload allowance estimate.

\begin{tabular}{|l|l|l|l|l|l|l|}
\hline \multicolumn{7}{|c|}{ Architecture option $\mathbf{A}^{\mathbf{2}}$} \\
\hline $\begin{array}{c}\text { GO Side } \\
\begin{array}{c}\text { Length } \\
(\mathbf{m})\end{array}\end{array}$ & $\begin{array}{c}\text { GO Mass } \\
\text { Range with } \\
\mathbf{7 . 5} \boldsymbol{\mu m} \text { film } \\
(\mathbf{k g})\end{array}$ & $\begin{array}{c}\text { GO Mass } \\
\text { Range with } \\
\mathbf{2 . 5} \boldsymbol{\mu m} \text { film } \\
(\mathbf{k g})\end{array}$ & $\begin{array}{c}\text { Platform } \\
\text { Mass range } \\
\text { with } \mathbf{7 . 5} \boldsymbol{\mu m} \\
\text { film } \mathbf{( k g )}\end{array}$ & $\begin{array}{c}\text { Platform } \\
\text { Mass range } \\
\text { with } \mathbf{2 . 5} \boldsymbol{\mu m} \\
\text { film } \mathbf{( k g )}\end{array}$ & $\begin{array}{c}\text { Payload } \\
\text { Mass with } \\
\mathbf{7 . 5} \boldsymbol{\mu m} \text { film } \\
(\mathbf{k g})\end{array}$ & $\begin{array}{c}\text { Payload } \\
\text { Mass with } \\
\mathbf{2 . 5} \boldsymbol{\mu m} \text { film } \\
(\mathbf{k g})\end{array}$ \\
\hline 50 & $120-107$ & $95-83$ & negative & negative & negative & negative \\
\hline 75 & $202-172$ & $146-116$ & $<12$ & $38-67$ & $<2$ & $8-13$ \\
\hline 100 & $306-255$ & $207-156$ & $21-71$ & $120-170$ & $4-14$ & $25-34$ \\
\hline 125 & $435-360$ & $281-205$ & $75-150$ & $229-305$ & $15-30$ & $46-61$ \\
\hline
\end{tabular}


Table 5 Summary of mission architecture option $\mathrm{A}+{ }^{2}$ mass allowance estimates, including ROM payload allowance estimate.

\begin{tabular}{|c|c|c|c|c|c|c|}
\hline \multicolumn{7}{|c|}{ Architecture option $\mathrm{A}+^{2}$} \\
\hline $\begin{array}{l}\text { GO Side } \\
\text { Length } \\
\text { (m) }\end{array}$ & $\begin{array}{c}\text { GO Mass } \\
\text { Range with } \\
7.5 \mu \mathrm{m} \text { film } \\
(\mathrm{kg})\end{array}$ & $\begin{array}{c}\text { GO Mass } \\
\text { Range with } \\
2.5 \mu \mathrm{m} \text { film } \\
(\mathrm{kg})\end{array}$ & $\begin{array}{c}\text { Platform } \\
\text { Mass range } \\
\text { with } 7.5 \mu \mathrm{m} \\
\text { film }(\mathrm{kg})\end{array}$ & $\begin{array}{c}\text { Platform } \\
\text { Mass range } \\
\text { with } 2.5 \mu \mathrm{m} \\
\text { film }(\mathrm{kg})\end{array}$ & $\begin{array}{c}\text { Payload } \\
\text { Mass with } \\
7.5 \mu \mathrm{m} \text { film } \\
(\mathrm{kg})\end{array}$ & $\begin{array}{c}\text { Payload } \\
\text { Mass with } \\
2.5 \mu \mathrm{m} \text { film } \\
(\mathrm{kg})\end{array}$ \\
\hline 50 & $120-107$ & $95-83$ & negative & negative & negative & negative \\
\hline 75 & $202-172$ & $146-116$ & negative & $<20$ & negative & $<4$ \\
\hline 100 & $306-255$ & $207-156$ & negative & $34-85$ & negative & $7-17$ \\
\hline 125 & $435-360$ & $281-205$ & $<17$ & $96-172$ & $<3$ & $19-34$ \\
\hline
\end{tabular}

Table 6 Summary of mission architecture option $\mathrm{B}^{2}$ mass allowance estimates, including ROM payload allowance estimate.

\begin{tabular}{|c|c|c|c|c|c|c|}
\hline \multicolumn{7}{|c|}{ Architecture option $\mathrm{B}^{2}$} \\
\hline $\begin{array}{l}\text { GO Side } \\
\text { Length } \\
\text { (m) }\end{array}$ & $\begin{array}{c}\text { GO Mass } \\
\text { Range with } \\
7.5 \mu \mathrm{m} \text { film } \\
(\mathrm{kg})\end{array}$ & $\begin{array}{c}\text { GO Mass } \\
\text { Range with } \\
2.5 \mu \mathrm{m} \text { film } \\
(\mathrm{kg})\end{array}$ & $\begin{array}{c}\text { Platform } \\
\text { Mass range } \\
\text { with } 7.5 \mu \mathrm{m} \\
\text { film }(\mathbf{k g})\end{array}$ & $\begin{array}{c}\text { Platform } \\
\text { Mass range } \\
\text { with } 2.5 \mu \mathrm{m} \\
\text { film }(\mathrm{kg})\end{array}$ & $\begin{array}{c}\text { Payload } \\
\text { Mass with } \\
7.5 \mu \mathrm{m} \text { film } \\
(\mathrm{kg})\end{array}$ & $\begin{array}{c}\text { Payload } \\
\text { Mass with } \\
2.5 \mu \mathrm{m} \text { film } \\
(\mathrm{kg})\end{array}$ \\
\hline 50 & $120-107$ & $95-83$ & negative & negative & negative & negative \\
\hline 75 & $202-172$ & $146-116$ & negative & $20-49$ & negative & $4-10$ \\
\hline 100 & $306-255$ & $207-156$ & $<39$ & $87-138$ & $<8$ & $17-28$ \\
\hline 125 & $435-360$ & $281-205$ & $24-100$ & $179-254$ & $5-20$ & $36-51$ \\
\hline
\end{tabular}

Table 7 Summary of mission architecture option $\mathrm{B}+{ }^{2}$ mass allowance estimates, including ROM payload allowance estimate.

\begin{tabular}{|c|c|c|c|c|c|c|}
\hline \multicolumn{7}{|c|}{ Architecture option $\mathbf{B}+{ }^{2}$} \\
\hline $\begin{array}{l}\text { GO Side } \\
\text { Length } \\
\text { (m) }\end{array}$ & $\begin{array}{c}\text { GO Mass } \\
\text { Range with } \\
7.5 \mu \mathrm{m} \text { film } \\
(\mathrm{kg})\end{array}$ & $\begin{array}{c}\text { GO Mass } \\
\text { Range with } \\
2.5 \mu \mathrm{m} \text { film } \\
(\mathrm{kg})\end{array}$ & $\begin{array}{c}\text { Platform } \\
\text { Mass range } \\
\text { with } 7.5 \mu \mathrm{m} \\
\text { film }(\mathrm{kg})\end{array}$ & $\begin{array}{c}\text { Platform } \\
\text { Mass range } \\
\text { with } 2.5 \mu \mathrm{m} \\
\text { film }(\mathrm{kg})\end{array}$ & $\begin{array}{c}\text { Payload } \\
\text { Mass with } \\
7.5 \mu \mathrm{m} \text { film } \\
(\mathrm{kg})\end{array}$ & $\begin{array}{c}\text { Payload } \\
\text { Mass with } \\
2.5 \mu \mathrm{m} \text { film } \\
(\mathrm{kg})\end{array}$ \\
\hline 50 & $120-107$ & $95-83$ & negative & negative & negative & negative \\
\hline 75 & $202-172$ & $146-116$ & negative & $<2$ & negative & $\sim 0$ \\
\hline 100 & $306-255$ & $207-156$ & negative & $4-54$ & negative & $1-11$ \\
\hline 125 & $435-360$ & $281-205$ & negative & $48-124$ & negative & $10-25$ \\
\hline
\end{tabular}

Table 8 Summary of mission architecture option $C^{2}$ mass allowance estimates, including ROM payload allowance estimate.

\begin{tabular}{|c|c|c|c|c|c|c|}
\hline \multicolumn{7}{|c|}{ Architecture option $\mathbf{C}^{2}$} \\
\hline $\begin{array}{c}\text { GO Side } \\
\text { Length } \\
\text { (m) }\end{array}$ & $\begin{array}{c}\text { GO Mass } \\
\text { Range with } \\
7.5 \mu \mathrm{m} \text { film } \\
(\mathrm{kg}) \\
\end{array}$ & $\begin{array}{c}\text { GO Mass } \\
\text { Range with } \\
2.5 \mu \mathrm{m} \text { film } \\
(\mathrm{kg})\end{array}$ & $\begin{array}{c}\text { Platform } \\
\text { Mass range } \\
\text { with } 7.5 \mu \mathrm{m} \\
\text { film }(\mathrm{kg})\end{array}$ & $\begin{array}{c}\text { Platform } \\
\text { Mass range } \\
\text { with } 2.5 \mu \mathrm{m} \\
\text { film }(\mathrm{kg})\end{array}$ & $\begin{array}{c}\text { Payload } \\
\text { Mass with } \\
7.5 \mu \mathrm{m} \text { film } \\
(\mathrm{kg}) \\
\end{array}$ & $\begin{array}{c}\text { Payload } \\
\text { Mass with } \\
2.5 \mu \mathrm{m} \text { film } \\
(\mathrm{kg})\end{array}$ \\
\hline 50 & $120-107$ & $95-83$ & negative & negative & negative & negative \\
\hline 75 & $202-172$ & $146-116$ & negative & $<26$ & negative & $<5$ \\
\hline 100 & $306-255$ & $207-156$ & negative & $45-96$ & negative & $9-19$ \\
\hline 125 & $435-360$ & $281-205$ & $<34$ & $113-188$ & $<7$ & $23-38$ \\
\hline
\end{tabular}


Table 9 Summary of mission architecture option $\mathrm{C}+{ }^{2}$ mass allowance estimates, including ROM payload allowance estimate.

\begin{tabular}{|c|c|c|c|c|c|c|}
\hline \multicolumn{7}{|c|}{ Architecture option $\mathrm{C}+{ }^{2}$} \\
\hline $\begin{array}{l}\text { GO Side } \\
\text { Length } \\
\text { (m) }\end{array}$ & $\begin{array}{c}\text { GO Mass } \\
\text { Range with } \\
7.5 \mu \mathrm{m} \text { film } \\
(\mathrm{kg})\end{array}$ & $\begin{array}{c}\text { GO Mass } \\
\text { Range with } \\
2.5 \mu \mathrm{m} \text { film } \\
(\mathrm{kg})\end{array}$ & $\begin{array}{c}\text { Platform } \\
\text { Mass range } \\
\text { with } 7.5 \mu \mathrm{m} \\
\text { film }(\mathrm{kg})\end{array}$ & $\begin{array}{c}\text { Platform } \\
\text { Mass range } \\
\text { with } 2.5 \mu \mathrm{m} \\
\text { film }(\mathrm{kg})\end{array}$ & $\begin{array}{c}\text { Payload } \\
\text { Mass with } \\
7.5 \mu \mathrm{m} \text { film } \\
(\mathrm{kg})\end{array}$ & $\begin{array}{c}\text { Payload } \\
\text { Mass with } \\
2.5 \mu \mathrm{m} \text { film } \\
(\mathrm{kg})\end{array}$ \\
\hline 50 & $120-107$ & $95-83$ & negative & negative & negative & negative \\
\hline 75 & $202-172$ & $146-116$ & negative & negative & negative & negative \\
\hline 100 & $306-255$ & $207-156$ & negative & $<17$ & negative & $<3$ \\
\hline 125 & $435-360$ & $281-205$ & negative & $<65$ & negative & $<13$ \\
\hline
\end{tabular}

\section{Analysis of Hybrid Solar Sail, Constant Acceleration Electric Propulsion Solar Polar Transfer}

As, in this scenario, the inclination as a function of time cannot be algebraically determined for a varying semimajor axis the effect of varying the out-of-plane thrust angle of the solar sail and/or constant acceleration electric propulsion in the first phase cannot be analytically generalized. However, as a conservative estimate of the transfer time to a polar orbit, $i=82.75^{\circ}$, both out-of-plane thrust angles in the first phase can be assumed as zero, allowing the transfer time to be determined simply as the sum of Eq. (43) and (45). It is found that the transfer time for such a hybrid propulsion system with a characteristic acceleration of $0.1 \mathrm{~mm} \mathrm{~s}^{-2}$, and a constant electric propulsion accelerations of $0.1 \mathrm{~mm} \mathrm{~s}^{-2}$, correspond to an initial thrust of $100 \mathrm{mN}$ for a $1000 \mathrm{~kg}$ spacecraft, to a polar orbit of target orbit radius of 0.48 au is approximately 13.84 years. In this trajectory only $16 \%$ of the transfer time, approximately 2.27 years, is the first phase where the orbit radius is being reduced from 1 au to 0.48 au, it is hence likely that the transfer time can be reduced by allowing one or both out-of-plane thrust angles in the first phase to be non-zero.

Using a special perturbations technique whereby modified equinoctial elements are used in the equations of motion, which are propagated using an explicit, variable step size Runge-Kutta $(4,5)$ formula, the Dormand-Price pair, a single-step method, ${ }^{28-30}$ the inclination at the end of phase 1 can be numerically determined. Hence, Table 10 shows the transfer time for a $1000 \mathrm{~kg}$ spacecraft with a hybrid constant acceleration (of $0.1 \mathrm{~mm} \mathrm{~s}$ ) electric propulsion and solar sail (characteristic accelerations of $0.1 \mathrm{~mm} \mathrm{~s}^{-2}$; giving a square sail side length of approximately $105 \mathrm{~m}$ ) propelled to a solar polar orbit, where the solar sail thrust is directed within the orbit plane, i.e. $\tilde{\chi}=0$, over a range of constant acceleration electric propulsion out-of-plane thrust angles. Note that within Table 10 (and Table 11) only the inclination at the end of phase 1 cannot be analytically determined. Furthermore, the second phase duration is determined using the general perturbations approximation in Eq. (45) as using the special perturbations solution would require the orbit to be circularized before beginning phase two to gain an accurate total time estimate. It is seen from Table 10 that the transfer time is approximately minimized, in this scenario, for a constant acceleration electric propulsion out-of-plane thrust angle, $\tilde{\chi} \cong 50^{\circ}$. Table 11 extends the results in Table 10 by fixing the constant acceleration electric propulsion out-of-plane thrust angle, $\tilde{\chi}=50^{\circ}$ and varying the solar sail out of plane thrust angle. From Table 11 it is seen that the transfer time to a solar polar orbit is approximately minimized, in this scenario, when the constant acceleration electric propulsion out of plane thrust angle, $\tilde{\chi} \cong 50^{\circ}$ and $\tilde{\gamma} \cong 60^{\circ}$; reducing the transfer time by approximately 1-year (7\%) from the conservative scenario presented in previously.

The results presented in Table 10 and Table 11 were manually generated in-order to gain an understanding of the solution space, from these results it is clear that the solution space is relatively simple, although a small local minimum is noted at around $\tilde{\chi} \cong 50^{\circ}$ and $\tilde{\gamma} \cong 45-50^{\circ}$. To extend Table 11 a simple numerical optimizer can thus be used to determine the best out-of-plane angles in phase 1 . As for Table 10 and Table 11 the second phase duration is determined using the general perturbations approximation in Eq. (45) and as such numerical integration is restricted to phase one, making the solution of the numerical optimization problem fast.

A Nelder-Mead Simplex Method, which does not use numerical or analytic gradients, ${ }^{31}$ is applied to solve an objective function that simply minimizes total duration with a solution tolerance on the control angles of $0.05^{\circ}$ and a solution tolerance of 1000 seconds on the trajectory duration. It is found that providing an initial guess where both angles are below the noted local minimum at around $\tilde{\chi} \cong 50^{\circ}$ and $\tilde{\gamma} \cong 45-50^{\circ}$ the optimiser converges to a local optimal solution in this vicinity. However, providing an initial guess where both angles are above the local minimum the optimizer determines a minimum trip time of 12.821 years using an electric propulsion thrust out of plane angle, $\tilde{\chi}=53.73^{\circ}$ and a solar sail out of plane angle of $\tilde{\gamma}=56.44^{\circ}$. 
Table 10 Hybrid transfer to a solar polar orbit for an in-plane solar sail thrust, i.e. $\widetilde{\gamma}=0$, and a range of constant acceleration electric propulsion out-of-plane thrust angles.

\begin{tabular}{|c|c|c|c|c|}
\hline $\begin{array}{c}\text { Out-of-plane angle in } \\
\text { phase one, } \tilde{\chi} \\
\text { (degrees) }\end{array}$ & $\begin{array}{c}\text { Inclination at end } \\
\text { of phase 1 } \\
\text { (degrees) }\end{array}$ & $\begin{array}{c}\text { Phase 1 } \\
\text { duration } \\
\text { (years) }\end{array}$ & Phase 2 duration & Total Duration \\
\hline 0 & 0.00 & 2.26 & 11.57 & 13.836 \\
\hline 10 & 1.33 & 2.28 & 11.39 & 13.670 \\
\hline 20 & 2.68 & 2.34 & 11.20 & 13.544 \\
\hline 30 & 4.14 & 2.46 & 10.99 & 13.452 \\
\hline 40 & 5.65 & 2.62 & 10.78 & 13.407 \\
\hline 45 & 6.45 & 2.72 & 10.67 & 13.395 \\
\hline 50 & 7.34 & 2.84 & 10.55 & 13.391 \\
\hline 55 & 8.19 & 3.00 & 10.43 & 13.424 \\
\hline 60 & 9.28 & 3.18 & 10.28 & 13.458 \\
\hline
\end{tabular}

Table 11 Hybrid transfer to a solar polar orbit for constant acceleration electric propulsion out of plane thrust angle, $\widetilde{\chi}=50^{\circ}$, and a range of solar sail thrust out-of-plane angles.

\begin{tabular}{|c|c|c|c|c|}
\hline $\begin{array}{c}\text { Out-of-plane angle in } \\
\text { phase one, } \tilde{\boldsymbol{\gamma}} \\
\text { (degrees) }\end{array}$ & $\begin{array}{c}\text { Inclination at end } \\
\text { of phase 1 } \\
\text { (degrees) }\end{array}$ & $\begin{array}{c}\text { Phase 1 } \\
\text { duration } \\
\text { (years) }\end{array}$ & Phase 2 duration & Total Duration \\
\hline 10 & 8.68 & 2.87 & 10.36 & 13.226 \\
\hline 20 & 10.08 & 2.94 & 10.16 & 13.103 \\
\hline 30 & 11.91 & 3.07 & 9.91 & 12.979 \\
\hline 40 & 13.72 & 3.26 & 9.65 & 12.915 \\
\hline 45 & 14.94 & 3.38 & 9.48 & 12.861 \\
\hline 50 & 15.98 & 3.53 & 9.34 & 12.864 \\
\hline 55 & 17.43 & 3.71 & 9.14 & 12.842 \\
\hline 60 & 18.91 & 3.90 & 8.93 & 12.830 \\
\hline 65 & 20.62 & 4.16 & 8.69 & 12.845 \\
\hline 70 & 22.43 & 4.44 & 8.44 & 12.874 \\
\hline
\end{tabular}

\section{Analysis of Hybrid Solar Sail, Constant Thrust Electric Propulsion Solar Polar Transfer}

As once again in this scenario the inclination as a function of time cannot be algebraically determined for a varying semi-major axis the effect of varying the out-of-plane thrust angle of the solar sail and/or constant thrust electric propulsion in the first phase cannot be analytically determined. As in the constant acceleration scenario, a conservative estimate of the transfer time to a polar orbit, $i=82.75^{\circ}$, can be gained by assuming both out-of-plane thrust angles in the first phase are zero, allowing the transfer time to be determined simply as the sum of Eq. (53) \& (55). The transfer time for such a hybrid propulsion system with a characteristic acceleration of $0.1 \mathrm{~mm} \mathrm{~s}^{-2}$, and a constant electric propulsion accelerations of $0.1 \mathrm{~mm} \mathrm{~s}^{-2}$, correspond to an initial thrust of $100 \mathrm{mN}$ for a $1000 \mathrm{~kg}$ spacecraft, to a polar orbit of target orbit radius of 0.48 au is approximately 10.4 years. Of this, 2.1 years ( 20\% of the transfer) is spent in phase 1, reducing the orbit radius from 1 au to 0.48 au. It is also of note that the transfer requires a solar sail of surface area $11020 \mathrm{~m}^{2}$ (equivalent to a square sail side length of $105 \mathrm{~m}$ ) and a fuel mass fraction of approximately $65 \%$, assuming a propulsion system exhaust velocity, $c$, of $50000 \mathrm{~m} / \mathrm{s}$, equivalent to a specific impulse of approximately $5097 \mathrm{~m} / \mathrm{s}$.

The out-of-plane angle analysis performed in the constant acceleration scenario is repeated for this constant thrust scenario. Once again, the Nelder-Mead Simplex Method is applied with an objective function to minimize total duration, with a solution tolerance on the control angles of $0.05^{\circ}$ and a solution tolerance of 1000 seconds on the trajectory duration. It is found that the optimizer determines a minimum trip time of 10.083 years using an electric propulsion thrust out of plane angle, $\tilde{\chi}=32.84^{\circ}$ and a solar sail out of plane angle of $\tilde{\gamma}=34.11^{\circ}$. 


\section{A. Strawman System Mass Budget}

Assuming two system launch masses of $1000 \mathrm{~kg}$ and $1600 \mathrm{~kg}$, where the latter is representative of the maximum possible Soyuz $C_{3}=0$ launch mass, a strawman system mass budget allocation can be developed using the models introduced in the earlier solar sail section. Furthermore, the use of QinetiQ T6 electric propulsion thrusters at full power, producing $143 \mathrm{mN}$ of thrust at specific impulse 4120 seconds, will be assumed a priori for this analysis. ${ }^{32}$ As such, the constant thrust electric propulsion system will provide an initial acceleration of $0.143 \mathrm{~mm} \mathrm{~s}^{-2}$ and $0.089 \mathrm{~mm} \mathrm{~s}^{-2}$, for the two assumed launch masses. The time/sail size required by the three principle mission architecture options introduced in Table 3, plus one of the fast mission architectures, is shown in Fig. 9 for the two system launch masses of $1000 \mathrm{~kg}$ and $1600 \mathrm{~kg}$.
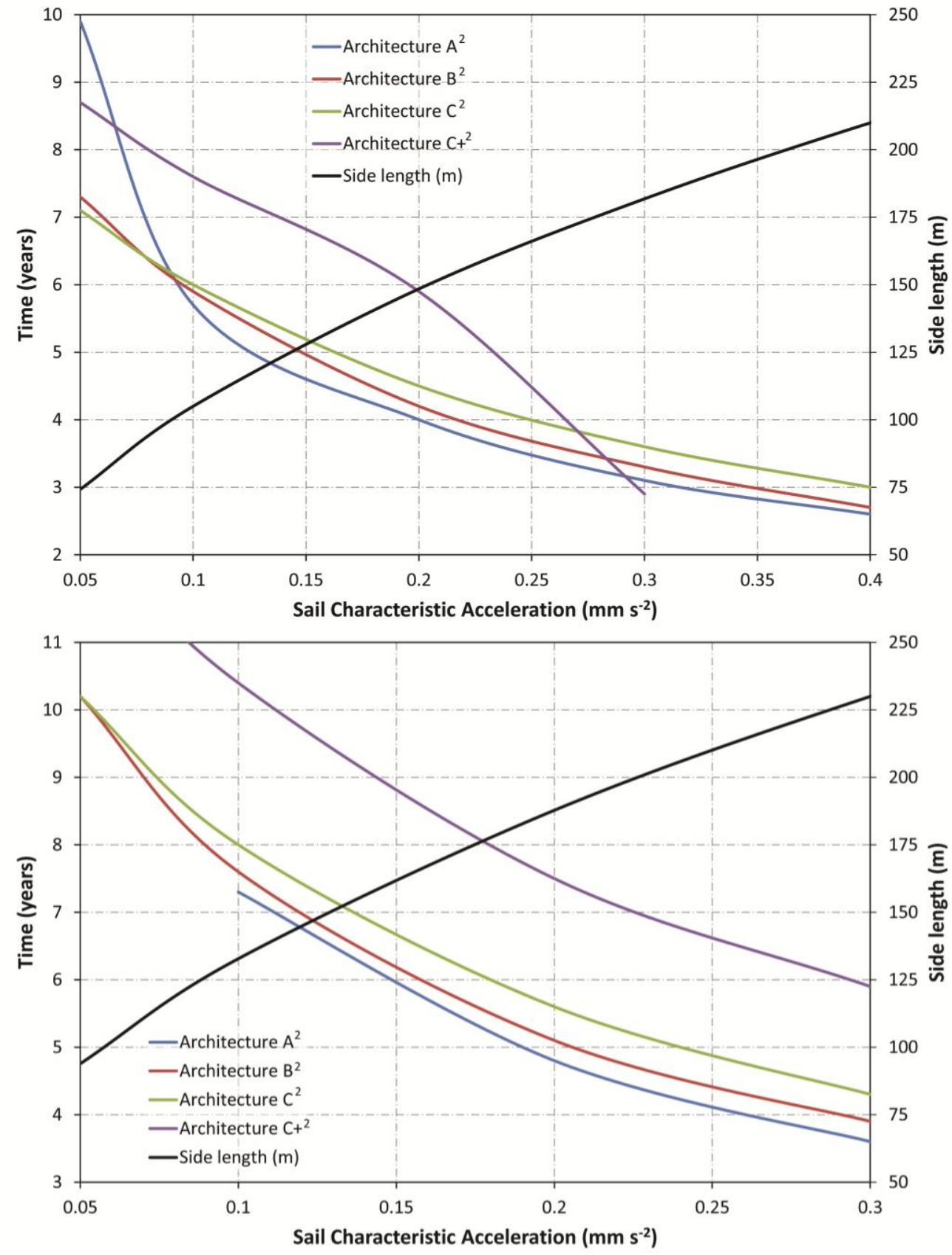

Fig. 9 Time, gossamer surface side length and characteristic acceleration for $1000 \mathrm{~kg}$ (top) and $1600 \mathrm{~kg}$ (bottom) hybrid propulsion spacecraft for mission architectures in Table 3.

American Institute of Aeronautics and Astronautics 
Considering mission architecture $\mathrm{B}^{2}$ using a $100 \mathrm{~m}$ gossamer structure it is possible to determine the approximately equivalent mission timeline for a non-hybrid mission, that is, a pure solar sail. Table 12 shows this comparison. Within Table 12 the spacecraft dry mass is also adjusted to account for the xenon tanks and other paraphernalia associated with the electric propulsion system. The adjustment is taken simply as $5 \%$ of the fuel mass and assumes that the power for the electric propulsion system was delivered using thin-film photovoltaic cells on the gossamer structure; however, the impact on surface reflectivity due to these cells is neglected in all analysis at this stage. From Table 12 it is seen that the hybrid system architecture offers real benefits for the higher launch mass where the trade is technology constrained, i.e. fixed sail size. This trade is again performed in Table 13 for architecture $\mathrm{C}^{2}$, where it is seen that the hybrid system architecture offers little, if any, advantage for either launch mass, and will most-likely result in a slower mission than a pure solar sail mission.

Alternatively to Table 12 and Table 13, a comparison can be made using the trip time; it is however noted from Fig. 9 that the $1600 \mathrm{~kg}$ launch mass scenario requires a gossamer structure in excess of $175 \mathrm{~m}$ in side-length to achieve a trip time of less than 5 -years, while the $1000 \mathrm{~kg}$ launch mass scenario requires a more modest $115 \mathrm{~m}$ and $125 \mathrm{~m}$ side length for architectures $\mathrm{A}^{2}$ and $\mathrm{B}^{2}$. Table 14 shows that for these time limited comparisons the hybrid system architectures do offers some advantages, in that the solar sail alone time is increased for the same sail, or the sail performance would need to increase to maintain the hybrid trip time. In summary, it can be concluded that for a mass constrained system hybridization of these propulsion systems is of less benefit than a thrust constrained system.

Table 12 Comparison of hybrid mission with a $100 \mathrm{~m}$ gossamer structure against a pure solar sail mission for architecture $\mathbf{B}^{2}$

\begin{tabular}{|l|l|l|}
\cline { 2 - 3 } \multicolumn{1}{l|}{} & $\mathbf{1 0 0 0} \mathbf{~ k g}$ & $\mathbf{1 6 0 0} \mathbf{~ k g}$ \\
\hline Time to target & $\mathbf{6 . 1}$ years & $\mathbf{8 . 0}$ years \\
\hline Fuel Mass Fraction & $68 \%$ & $56 \%$ \\
\hline Fuel Mass & $681 \mathrm{~kg}$ & $894 \mathrm{~kg}$ \\
\hline Dry Spacecraft Mass (inc. gossamer structure) & $319 \mathrm{~kg}$ & $706 \mathrm{~kg}$ \\
\hline Dry Spacecraft Characteristic Acceleration & $0.293 \mathrm{~mm} \mathrm{~s}^{-2}$ & $0.132 \mathrm{~mm} \mathrm{~s}^{-2}$ \\
\hline Dry Spacecraft Lightness Number & 0.0494 & 0.0223 \\
\hline Time to target of sail alone & 7.4 years & 11.7 years \\
\hline Adjusted Dry Spacecraft Mass (inc. gossamer structure) & $285 \mathrm{~kg}$ & $661 \mathrm{~kg}$ \\
\hline Adjusted Dry Spacecraft Characteristic Acceleration & $0.327 \mathrm{~mm} \mathrm{~s}^{-2}$ & $0.141 \mathrm{~mm} \mathrm{~s}^{-2}$ \\
\hline Adjusted Dry Spacecraft Lightness Number & 0.0551 & 0.0238 \\
\hline Adjusted time to target of sail alone & $\mathbf{4 . 7}$ years & $\mathbf{1 1 . 0}$ years \\
\hline
\end{tabular}

Table 13 Comparison of hybrid mission with a $100 \mathrm{~m}$ gossamer structure against a pure solar sail mission for architecture $\mathbf{C}^{2}$

\begin{tabular}{|l|l|l|}
\cline { 2 - 3 } \multicolumn{1}{c|}{} & $\mathbf{1 0 0 0} \mathbf{~ k g}$ & $\mathbf{1 6 0 0} \mathbf{~ k g}$ \\
\hline Time to target & $\mathbf{6 . 1}$ years & $\mathbf{9 . 8}$ years \\
\hline Fuel Mass Fraction & $68 \%$ & $68 \%$ \\
\hline Fuel Mass & $681 \mathrm{~kg}$ & $1095 \mathrm{~kg}$ \\
\hline Dry Spacecraft Mass (inc. gossamer structure) & $319 \mathrm{~kg}$ & $505 \mathrm{~kg}$ \\
\hline Dry Spacecraft Characteristic Acceleration & $0.293 \mathrm{~mm} \mathrm{~s}^{-2}$ & $0.185 \mathrm{~mm} \mathrm{~s}^{-2}$ \\
\hline Dry Spacecraft Lightness Number & 0.0494 & 0.0312 \\
\hline Time to target of sail alone & 6.2 years & 9.9 years \\
\hline Adjusted Dry Spacecraft Mass (inc. gossamer structure) & $285 \mathrm{~kg}$ & $450 \mathrm{~kg}$ \\
\hline Adjusted Dry Spacecraft Characteristic Acceleration & $0.238 \mathrm{~mm} \mathrm{~s}^{-2}$ & $0.208 \mathrm{~mm} \mathrm{~s}^{-2}$ \\
\hline Adjusted Dry Spacecraft Lightness Number & 0.0553 & 0.0351 \\
\hline Adjusted time to target of sail alone & $\mathbf{5 . 6}$ years & $\mathbf{8 . 8}$ years \\
\hline
\end{tabular}


Table 14 Comparison of time limited hybrid missions for architecture $\mathrm{A}^{2}$ and $\mathrm{B}^{2}$.

\begin{tabular}{|l|l|l|}
\hline Mission Architecture & \multicolumn{1}{|c|}{$\mathbf{A}^{\mathbf{2}}$} & \multicolumn{1}{c|}{$\mathbf{B}^{\mathbf{2}}$} \\
\hline Time to target & $\mathbf{5}$ years & $\mathbf{5}$ years \\
\hline Fuel Mass Fraction & $56 \%$ & $56 \%$ \\
\hline Fuel Mass & $558 \mathrm{~kg}$ & $558 \mathrm{~kg}$ \\
\hline Dry Spacecraft Mass (inc. gossamer structure) & $442 \mathrm{~kg}$ & $442 \mathrm{~kg}$ \\
\hline Dry Spacecraft Characteristic Acceleration & $0.211 \mathrm{~mm} \mathrm{~s}^{-2}$ & $0.211 \mathrm{~mm} \mathrm{~s}$ \\
\hline Dry Spacecraft Lightness Number & 0.0356 & 0.0356 \\
\hline Time to target of sail alone & 6.7 years & 7.3 years \\
\hline Adjusted Dry Spacecraft Mass (inc. gossamer structure) & $414 \mathrm{~kg}$ & $414 \mathrm{~kg}$ \\
\hline Adjusted Dry Spacecraft Characteristic Acceleration & $0.226 \mathrm{~mm} \mathrm{~s}^{-2}$ & $0.226 \mathrm{~mm} \mathrm{~s}^{-2}$ \\
\hline Adjusted Dry Spacecraft Lightness Number & 0.0380 & 0.0380 \\
\hline Adjusted time to target of sail alone & $\mathbf{6 . 3}$ years & $\mathbf{6 . 9}$ years \\
\hline
\end{tabular}

\section{Conclusion}

It has been shown that orbit averaging techniques can be used to develop an analytical approximation of a circleto-circle low-thrust trajectory transfer with plane-change. Analytical expressions were developed for constant acceleration and thrust electric propulsion, and solar sail propulsion. It was found that electric propulsion required an excessive fuel mass fraction for transfers to solar polar orbits, while the solar sail expression was accurate to within $1 \%$ of the time-optimal solution obtained through numerical optimization. It was also found that the structure of the three transfers was significantly different; a constant acceleration electric propulsion transfer is optimized by performing the complete plane-change at the maximum circular orbit radius, while a constant thrust electric propulsion transfer is optimized by performing the complete plane-change at the minimum circular orbit radius. It was analytically demonstrated however that the optimal solar sail transfer combines plane-change and the change in orbit radius, and furthermore, the optimal level of plane change was analytically determined.

Expressions were also developed for spacecraft with hybrid electric (constant acceleration and thrust) and solar sail propulsion. However, no analytical expression could be derived for the change of semi-major axis as a function of time, whilst also changing the inclination; as such the change in inclination could not be analytically quantified, which in-turn prohibited the development of a fully analytical expression for such hybrid propulsion systems. The use of a simple Nelder-Mead Simplex Method however allowed this to be easily overcome and it was found that the optimal hybrid electric (constant acceleration and thrust) and solar sail propulsion transfer was similar in basic structure to that of a solar sail.

Finally, a strawman system design analysis was performed using the developed analytical expressions. It was determined that, assuming an initially circular orbit at 1 au within the ecliptic plane, that the minimum radius is the target radius, that no gravity-assists are used, and that the transfer is limited to 5-years that a sail characteristic acceleration of less than $0.5 \mathrm{~mm} / \mathrm{s}^{2}$ can attain an orbit that maintains the observer-to-solar pole zenith angle below 40 degrees for 25 days; the approximate sidereal rotation period of the Sun, based on a solar latitude of 16 degrees. However, a sail characteristic acceleration of more than $0.5 \mathrm{~mm} / \mathrm{s}^{2}$ is required to maintain the observer-to-solar pole zenith angle below 30 degrees for 25 days. It was also found that the hybridization of electric propulsion and solar sail propulsion was, typically, of more benefit when the system was thrust constrained than when it was mass constrained.

\section{Acknowledgments}

The work presented herein was conducted with the support of the European Space Agency.

\section{References}

${ }^{1}$ Wright, J.L. and Warmke, J.M., "Solar Sail Mission Applications", Paper 76-808, AIAA/AAS Astrodynamics Conference, San Diego, California, August 18-20, 1976.

${ }^{2}$ Sauer, C. G., Jr., "Solar Sail Trajectories for Solar-Polar and Interstellar Probe Missions," AAS 99-336, Proceedings of AAS/AIAA Astrodynamics Specialists Conference, Girdwood, Alaska, August 1999.

${ }^{3}$ Goldstein, B., Buffington, A., Cummings, A.C., Fisher, R., Jackson, B.V., Liewer, P.C., Mewaldt, R.A., Neugebauer, M., "A Solar Polar Sail Mission: Report of a Study to Put a Scientific Spacecraft in a Circular Polar 
Orbit about the Sun", Proceedings of MTG: SPIE International Symposium on Optical Science, Engineering and Instrumentation, San Diego, California, July 1998.

${ }^{4}$ Macdonald, M., Hughes, G.W., M'Innes, C.R., Lyngvi, A., Falkner, P., Atzei, A. "Solar polar orbiter: a solar sail technology reference study", Journal of Spacecraft and Rockets, Vol. 43 No. 5, pp. 960-972, 2006.

${ }^{5}$ Macdonald, M., McInnes, C.R., "Solar Sail Mission Applications and Advancement", Advances in Space Research, Vol. 48, Iss. 11, pp. 1702 - 1716, 2011.

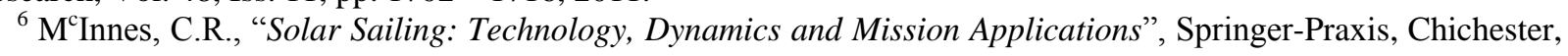
1999.

${ }^{7}$ Mooij, E., Noomen, R., Candy, S., "Evolutionary Optimization for a Solar Sailing Solar Polar Mission" AIAA 2006-6180, AIAA/AAS Astrodynamics Specialist Conference and Exhibit, Keystone, Colorado, August 21 - 24, 2006.

${ }^{8}$ Dachwald, B., Ohndorf, A., Wie, B., "Solar Sail Trajectory Optimization for the Solar Polar Imager (SPI) Mission", AIAA 2006-6177, AIAA/AAS Astrodynamics Specialist Conference and Exhibit, Keystone, Colorado, August $21-24,2006$.

${ }^{9}$ Mengali, G., Quarta, A.A., "Solar Sail Near-Optimal Circular Transfers with Plane Change", Journal of Guidance, Control and Dynamics, Vol. 32, No. 2, pp. 456 - 463, 2009.

${ }^{10}$ Quarta, A.A., Mengali, G., "Approximate Solutions to Circle-to-Circle Solar Sail Orbit Transfers”, Journal of Guidance, Control and Dynamics, In Press, 2013.

${ }^{11}$ Dachwald, B., Mengali, G., Quarta, A.A., Macdonald, M., "Parametric model and optimal control of solar sails with optical degradation", Journal of Guidance, Control and Dynamics, Vol. 29, No. 5, pp. 1170 - 1178, 2006.

${ }^{12}$ Dachwald, B., Macdonald, M., M ${ }^{\mathrm{c}}$ Innes, C.R., Mengali, G., "Impact of optical degradation on solar sail mission performance", Journal of Spacecraft and Rockets, Vol. 44, No. 4, pp. 740 - 749, 2007.

${ }^{13}$ Appourchaux, T., Liewer, P., Watt, M., Alexander, D., Andretta, V., Auchere, F., D'Arrigo, P., Ayon, J., Corbard, T., Fineschi, S., Finsterle, W., Floyd, L., Garbe, G., Gizon, L., Hassler, D., Harra, L., Kosovichev, A., Leibacher, J., Leipold, M., Murphy, N., Maksimovic, M., Martinez-Pillet, V., Matthews, BSA., Mewaldt, R., Moses, D., Newmark, J., Regnier, S., Schmutz, W., Socker, D., Spadaro, D., Stuttard, M., Trosseille, C., Ulrich, R., Velli, M., Vourlidas, A., Wimmer-Schweingruber, C.R., Zurbuchen, T., "POLAR Investigation of the Sun - POLARIS", Experimental Astronomy, Vol. 23, pp. 1079 - 1117, 2009.

${ }^{14}$ Vallado, D., "Fundamentals of Astrodynamics and Applications", Microcosm Press, Hawthorne, CA, 2007, pp. $377-383$.

${ }^{15}$ Roy, A.E., “Orbital Motion”, Taylor \& Francis Group, LLC, Abingdon, Oxon, U.K., 2005, pp. 208 - 220.

${ }^{16}$ Wakker, K.E., "Rocket Propulsion and Spaceflight Dynamics", Pitman, London, 1984, pp. 462 - 479.

${ }^{17}$ Wiesel, W.E., "Spaceflight Dynamics", McGraw-Hill, New York, 1991, pp. 89 - 91.

${ }^{18}$ Macdonald M., M'Innes C. R., "Analytic Control Laws for Near-Optimal Geocentric Solar Sail Transfers", AAS 01-472, Advances in the Astronautical Sciences, Vol. 109, No. 3, pp. 2393-2411, 2001.

${ }^{19}$ Macdonald M., M'Innes C. R., "Analytical Control Laws for Planet-Centered Solar Sailing”, Journal of Guidance, Control, and Dynamics, Vol. 28, No. 5, pp. 1038-1048, 2005.

${ }^{20}$ Macdonald M., M'Innes C. R., Dachwald, B., "Heliocentric Solar Sail Orbit Transfers with Locally Optimal Control Laws", Journal of Spacecraft and Rockets, Vol. 44, No. 1, pp 273 - 276, 2007.

${ }^{21}$ Sickinger, C., Herbeck, L., "Deployment Strategies, Analysis and Tests for the CFRP Booms of a Solar Sail", In proceedings of European Conference on Spacecraft Structures, Materials and Mechanical Testing Conference, CNES, Toulouse, France, 2002.

${ }^{22}$ Herbeck, L., Sickinger, C., Eiden, M., Leipold, M., "Solar Sail hardware Developments", In proceedings of European Conference on Spacecraft Structures, Materials and Mechanical Testing Conference, CNES, Toulouse, France, 2002.

${ }^{23}$ Murphy, D.M., M'Eachen, M.E., Macy, B.D., gasper, J.L. "Demonstration of a 20-m Solar Sail System”, AIAA 2005-2126, 46 ${ }^{\text {th }}$ AIAA/ASME/ASCE/AHS/ASC Structures, Structural Dynamics \& Materials Conference, Austin, Texas, 2005.

${ }^{24}$ Lichodziejewshi, D., Derbès, B., "Vacuum Deployment and Testing of a 4-Quadrant Scaleable Inflatable Rigidizable Solar Sail System", AIAA 2005-2122, 46 ${ }^{\text {th }}$ AIAA/ASME/ASCE/AHS/ASC Structures, Structural Dynamics \& Materials Conference, Austin, Texas, 2005.

${ }^{25}$ Sleight D.W., Michii, Y., Lichodziejewshi, D., Derbès, B., Mann, T.O., "Structural Analysis of an Inflation Deployment Solar Sail with Experimental Validation", AIAA 2005-3727, 41 ${ }^{\text {st }}$ AIAA/ASME/SAE/ASEE Joint Propulsion Conference \& Exhibit, Tucson, Arizona, 2005. 
${ }^{26}$ Mann, T., Behun, V., Lichodziejewshi, D., Derbès, B., Sleight, D., "Ground Testing a 20-meter Inflation Deployed Solar Sail", AIAA 2006-1707, $47^{\text {th }}$ AIAA/ASME/ASCE/AHS/ASC Structures, Structural Dynamics \& Materials Conference, Newport, Rhode Island, 2006.

${ }^{27}$ Wertz, J. R., Larson, W. J., "Space Mission Analysis and Design", $3^{\text {rd }}$ Edition, Microcosm Press, El Segundo, California, October 1999.

${ }^{28}$ Giacaglia, G.E.O., "The Equations of Motion of an Artificial Satellite in Nonsingular Variables", Celestial Mechanics, Vol. 15, pp. 191-215, 1977.

${ }^{29}$ Walker, M.J.H., Ireland, B., Owens, J., “A Set of Modified Equinoctial Elements”, Celestial Mechanics, Vol. 36, pp. 191-215, 1985.

${ }^{30}$ Dormand, J.R., Price, P.J., “A Family of Embedded Runge-Kutta Formulae”, Journal of Computing and Applied Mathematics, Vol. 6, pp 19-26, 1980.

${ }^{31}$ Lagarias, J.C., Reeds, J. A., Wright, M. H., Wright, P. E., "Convergence Properties of the Nelder-Mead Simplex Method in Low Dimensions", SIAM Journal of Optimization, Vol. 9 Number 1, pp. 112-147, 1998.

${ }^{32}$ Gessini, P., Gabriel, S.B., Fearn, D.G., "Thrust Characterization of a T6 Hollow Cathode", IEPC-05-257, Proceedings of the $29^{\text {th }}$ International Electric Propulsion Conference, Princeton University, New Jersey, October/November, 2005. 